Anton Bovier · Véronique Gayrard · Markus Klein

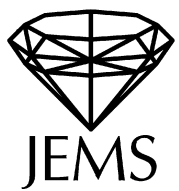

\title{
Metastability in reversible diffusion processes II. Precise asymptotics for small eigenvalues
}

Received September 15, 2002 and in revised form February 27, 2004

\begin{abstract}
We continue the analysis of the problem of metastability for reversible diffusion processes, initiated in [BEGK3], with a precise analysis of the low-lying spectrum of the generator. Recall that we are considering processes with generators of the form $-\epsilon \Delta+\nabla F(\cdot) \nabla$ on $\mathbb{R}^{d}$ or subsets of $\mathbb{R}^{d}$, where $F$ is a smooth function with finitely many local minima. Here we consider only the generic situation where the depths of all local minima are different. We show that in general the exponentially small part of the spectrum is given, up to multiplicative errors tending to one, by the eigenvalues of the classical capacity matrix of the array of capacitors made of balls of radius $\epsilon$ centered at the positions of the local minima of $F$. We also get very precise uniform control on the corresponding eigenfunctions. Moreover, these eigenvalues can be identified with the same precision with the inverse mean metastable exit times from each minimum. In [BEGK3] it was proven that these mean times are given, again up to multiplicative errors that tend to one, by the classical Eyring-Kramers formula.
\end{abstract}

Keywords. Metastability, diffusion processes, spectral theory, potential theory, capacity, exit times

\section{Introduction}

In this paper we continue the investigation of reversible diffusion processes initiated in [BEGK3]. Recall that we are interested in processes $X_{\epsilon}(t)$ that are given as solutions of an Itô stochastic differential equation

$$
d X_{\epsilon}(t)=-\nabla F\left(X_{\epsilon}(t)\right) d t+\sqrt{2 \epsilon} d W(t)
$$

Work of A. Bovier partially supported by the DFG Research Center FZT 86, of V. Gayrard by the FNS under contract No. FN-21-65267.01, and of M. Klein by Sfb 288.

A. Bovier: Weierstrass-Institut für Angewandte Analysis und Stochastik, Mohrenstrasse 39, 10117 Berlin, and Institut für Mathematik, Technische Universität Berlin, Strasse des 17. Juni 136, 10623 Berlin, Germany; e-mail: bovier@wias-berlin.de

V. Gayrard: EPFL,FSB,SMA,IMB, 1015 Lausanne, Switzerland; present address: Centre de Physique Théorique, CNRS, Luminy, Case 907, F-13288 Marseille, Cedex 9, France

M. Klein: Institut für Mathematik, Universität Potsdam, Am Neuen Palais 10, D-14469 Potsdam, Germany; e-mail: mklein@math.uni-potsdam.de

Mathematics Subject Classification (2000): 82C44, 60K35 
on a regular domain $\Omega \subseteq \mathbb{R}^{d}$, where the drift $\nabla F$ is generated by a potential function that is sufficiently regular, and that both $F(x)$ and $\|\nabla F(x)\|$ tend to $\infty$ at infinity. We are interested in the case when the function $F(x)$ has several local minima. We always assume that $X_{\epsilon}$ is killed on $\Omega^{c}$ if it exists.

For a general introduction to the topic and its history we refer to the introduction of [BEGK3]. In that paper we have studied the so-called metastable exit times from attractors of local minima of $F$ and we have given a precise asymptotic estimate for the mean value of these times. These estimates were in turn based on precise estimates of certain Newtonian capacities of sets containing small balls centered at the locations of the minima of $F$.

In the present paper we turn to the investigation of the low-lying spectrum of the generators of the process defined by $[1.1$, , i.e.

$$
L_{\epsilon} \equiv-\epsilon \Delta+\nabla F(x) \cdot \nabla
$$

with Dirichlet boundary conditions on $\Omega^{c}$ (if $\Omega \neq \mathbb{R}^{d}$ ). Under our assumptions on $F$, given below, the spectrum of this operator will be discrete. Moreover, it is well known that the spectrum of such operators has precisely one exponentially small eigenvalue for each local minimum of the function $F$, and more or less rough estimates of their precise values are known [FW] Ma, Mi]. Wentzell [W2] and Freidlin and Wentzell [FW] obtain an estimate for the exponential rate, i.e. they identify $\lim _{\epsilon \downarrow 0} \epsilon^{-1} \ln \lambda_{i}(\epsilon)$ using large deviation methods. Sharper estimates, with multiplicative errors of order $\epsilon^{ \pm k d}$, were obtained for principal eigenvalues by Holley, Kusuoka, and Strook [HKS] using a variational principle; these methods were extended to the full set of exponentially small eigenvalues by Miclo [Mi] (see also [Ma]).

Our purpose here is to get sharp estimates, i.e. we seek upper and lower bounds with multiplicative errors that tend to one as $\epsilon$ tends to zero. Such estimates are known in the one-dimensional case (see e.g. [BuMa1, BuMa2] and references therein), whereas in the multi-dimensional case only heuristic results based on formal power series expansions of WKB type exist (see e.g. [Kolo] for an analysis of the situation). This is due to the fact that the stochastic tunneling problem leads to a particularly degenerate form of the general tunnelling problem in the context of Schrödinger operators in which classical results [Si1, Si2, [HS1. [HS4] are not immediately applicable. While the methods introduced in the third paper on quantum mechanical tunneling by Helffer and Sjöstrand [HS3] should in principle allow one to justify such expansions, their implementation seems rather tedious and has not been carried out to our knowledge 1

Here we will resort to a different approach that combines ideas already suggested in [W1] with potential-theoretic ideas. In fact, this approach was developed in [BEGK2] in the setting of discrete Markov chains, where indeed many technical problems we will be facing here disappear, and that may serve as a nice introduction.

\footnotetext{
1 After submission of this paper, B. Helffer realised that under stronger regularity assumptions $\left(C^{\infty}\right)$ complete asymptotic expansions of the low-lying eigenvalues should be obtainable using methods developed in [HS4] in the context of the Witten complex. This was then carried out in $[\mathrm{HN}]$ in the case of a two-well potential, and the treatment of the general case is in progress [HKN].
} 
We will work under the same assumptions on $F$ as in [BEGK3] throughout this paper, namely:

\section{Assumptions (H.1)}

(i) $F \in C^{3}(\Omega), \Omega \subseteq \mathbb{R}^{d}$ open and connected.

(ii) If $\Omega$ is unbounded, then

(ii.1) $\liminf _{x \rightarrow \infty}|\nabla F(x)|=\infty$, and

(ii.2) $\liminf _{x \rightarrow \infty}(|\nabla F(x)|-2 \Delta F(x))=\infty$.

For any two sets $A, B \subset \Omega$, define the height of the saddle between $A$ and $B$ by

$$
\widehat{F}(A, B) \equiv \inf _{\omega: \omega(0) \in A, \omega(1) \in B} \sup _{t \in[0,1]} F(\omega(t)),
$$

where the infimum is over all continuous paths $\omega$ in $\Omega$.

Remark. Condition (H.1) ensures that the resolvent of the generator $L_{\epsilon}$ is compact for $\epsilon$ sufficiently small. Moreover, it implies that $F$ has exponentially tight level sets in the sense that for all $a \in \mathbb{R}$,

$$
\int_{y: F(y) \geq a} e^{-F(y) / \epsilon} d y \leq C e^{-a / \epsilon},
$$

where $C=C(a)<\infty$ is uniform in $\epsilon \leq 1$.

In the following, the notion of saddle points of $F$ will be crucial. The set of saddle points is intuitively the subset of the set $\mathcal{G}(A, B)=\{z: F(z)=\widehat{F}(A, B)\}$ that cannot be avoided by any paths $\omega$ that try to stay as low as possible. In general we have to define this set as follows:

Definition 1.1. Let $\mathcal{P}(A, B)$ denote the set of minimal paths from $A$ to $B$,

$$
\mathcal{P}(A, B) \equiv\left\{\omega \in C([0,1], \Omega): \omega(0) \in A, \omega(1) \in B, \sup _{t \in[0,1]} F(\omega(t))=\widehat{F}(A, B)\right\}
$$

$A$ gate $G(A, B)$ is a minimal subset of $\mathcal{G}(A, B)$ with the property that all minimal paths intersect $G(A, B)$. Note that $G(A, B)$ is in general not unique. Then the set $\mathcal{S}(A, B)$ of saddle points is the union over all gates $G(A, B)$.

To avoid complications that are not our main concern here, we will make the general assumption that all saddle points we will deal with are non-degenerate in the following sense:

\section{Assumption (ND)}

(o) The set, $\mathcal{M}$, of local minima of $F$ is finite and for any two local minima $x, y \in \mathcal{M}$, the set $G(x, y)$ is uniquely defined and consists of a finite set of isolated points $z_{i}^{*}(x, y)$.

(i) The Hessian matrix of $F$ at all local minima $x_{i} \in \mathcal{M}$ and all saddle points $z_{i}^{*}$ is non-degenerate (i.e. has only non zero-eigenvalues). 
When dealing with domains $\Omega$ with non-empty boundary we will encounter situations where saddle points in $\partial \Omega$ are relevant. While this does not lead to serious problems per se, there appears rather naturally a great variety of cases that makes the formulation of general results rather cumbersome. We prefer to avoid having to discuss these issues by dealing exclusively with situations in which the boundary is never reached by the process, i.e. we make the further

Assumption (IB). For any sequence of points $x_{i} \in \Omega$ such that $\lim _{i \uparrow \infty} x_{i} \in \partial \Omega$, $\lim _{i \uparrow \infty} F\left(x_{i}\right)=\infty$.

Our main interest is in the distribution of stopping times

$$
\tau_{A} \equiv \inf \{t>0: X(t) \in A\}
$$

for the process starting at one minimum, say $x \in \mathcal{M}$, of $F$, when $A=B_{\rho}(y)$ is a small ball of radius $\rho$ around another minimum, $y \in \mathcal{M}$. It will actually become apparent that the precise choice of the hitting set is often not important, and that the problem is virtually equivalent to considering the escape from a suitably chosen neighborhood of $x$, provided this neighborhood contains the relevant saddle points connecting $x$ and $y$.

Let us now state the main results of this paper.

Given two disjoint closed sets $A, D$, we will denote by $h_{A, D}(x)$ the equilibrium potential, by $e_{A, D}(d y)$ the equilibrium measure, and by $\operatorname{cap}_{A}(D)$ the Newtonian capacity corresponding to the Dirichlet problem with boundary conditions 1 on $A$ and 0 on $D$. The precise definitions of these classical quantities (see e.g. [BluGet, Doo, Szni]) are recalled in Section 2 of [BEGK3].

Theorem 1.2. Assume that $F$ has $n$ local minima, $x_{1}, \ldots, x_{n}$, and that for some $\theta>0$ the minima $x_{i}$ of $F$ can be labeled in such a way that, with $\mathcal{M}_{k} \equiv\left\{x_{1}, \ldots, x_{k}\right\}$ and $\mathcal{M}_{0} \equiv \Omega^{c}$

$$
F\left(z^{*}\left(x_{k}, \mathcal{M}_{k-1}\right)\right)-F\left(x_{k}\right) \leq \min _{i<k}\left(F\left(z^{*}\left(x_{i}, \mathcal{M}_{k} \backslash x_{i}\right)\right)-F\left(x_{i}\right)\right)-\theta
$$

for all $k=1, \ldots, n$. Set $B_{i} \equiv B_{\epsilon}\left(x_{i}\right), \mathcal{S}_{k} \equiv \bigcup_{i=1}^{k} B_{i}$, and $h_{k}(y) \equiv h_{B_{k}, \mathcal{S}_{k-1}}(y)$. Assume moreover that all saddle points $z^{*}\left(x_{k}, \mathcal{M}_{k-1}\right)$ are unique, and that the Hessian of $F$ is non-degenerate at all these saddle points and at all local minima. Then there exists $\delta>0$ such that the $n$ exponentially small eigenvalues $\lambda_{1}<\cdots<\lambda_{n}$ of $L_{\epsilon}$ satisfy:

$$
\lambda_{1}=0
$$

and for $k=2, \ldots, n$,

$$
\begin{aligned}
\lambda_{k}= & \frac{\operatorname{cap}_{B_{k}}\left(\mathcal{S}_{k-1}\right)}{\left\|h_{k}\right\|_{2}^{2}}\left(1+O\left(e^{-\delta / \epsilon}\right)\right)=\frac{1}{\mathbb{E}_{x_{k}} \tau_{\mathcal{S}_{k-1}}}\left(1+O\left(e^{-\delta / \epsilon}\right)\right) \\
= & \frac{\left|\lambda_{1}^{*}\left(z^{*}\left(x_{k}, \mathcal{M}_{k-1}\right)\right)\right|}{2 \pi} \sqrt{\frac{\operatorname{det}\left(\nabla^{2} F\left(x_{k}\right)\right)}{\left|\operatorname{det}\left(\nabla^{2} F\left(z^{*}\left(x_{k}, \mathcal{M}_{k-1}\right)\right)\right)\right|}} e^{-\left[F\left(z^{*}\left(x_{k}, \mathcal{M}_{k-1}\right)\right)-F\left(x_{k}\right)\right] / \epsilon} \\
& \times\left(1+O\left(\epsilon^{1 / 2}|\ln \epsilon|\right)\right),
\end{aligned}
$$

where $\lambda_{1}^{*}\left(z^{*}\right)$ denotes the unique negative eigenvalue of the Hessian of $F$ at the saddle point $z^{*}$. 
Remark. The theorem can be seen as containing three results: First, an asymptotically sharp identification of the exponentially small eigenvalues with the inverse mean exit times from local minima; this is a general feature of metastable systems (see e.g. [D1, D2, D3, GS, GM] for earlier results). Second, it relates these eigenvalues precisely to Newtonian capacities; this is the key difference from our results to e.g. the approach of Kolokoltsov and Makarov [KoMak, Kolo], since it allows thirdly to get an explicit expression for the eigenvalues in terms of the potential $F$.

Remark. Conditions (1.7) state that "all valleys of $F$ have different depths", which is in some sense the generic situation. In this case a number of simplifications take place, in particular we do not have to deal with degenerate eigenvalues. These conditions are completely analogous to the conditions imposed in [BEGK2]. Our general approach does, however, in principle also allow us to treat degenerate situations. We postpone the treatment of such cases to future work.

In the course of the proof of Theorem 1.2 we will also gain rather detailed control on the eigenfunctions of $L_{\epsilon}$ corresponding to its small eigenvalues.

Theorem 1.3. Under the assumptions of Theorem 1.2 if $\phi_{k}$ denotes the normalized eigenfunction corresponding to the eigenvalue $\lambda_{k}$, then there exists $\delta>0$ such that

$$
\begin{aligned}
\phi_{k}(y) & =\frac{h_{B_{\epsilon}\left(x_{k}\right), \mathcal{S}_{k-1}}(y)}{\left\|h_{B_{\epsilon}\left(x_{k}\right), \mathcal{S}_{k-1}}\right\|_{2}}\left(1+O\left(e^{-\delta / \epsilon}\right)\right)+O\left(e^{-\delta / \epsilon}\right), \\
\text { where }_{B_{\epsilon}\left(x_{k}\right), \mathcal{S}_{k-1}}(y) & =\mathbb{P}_{y}\left[\tau_{B_{\epsilon}\left(x_{k}\right)}<\tau_{\mathcal{S}_{k-1}}\right] .
\end{aligned}
$$

Remark. We give even more precise expressions for the eigenfunctions in the course of the proofs later on. Note that there is considerable interest in the knowledge of eigenfunctions in the context of numerical schemes designed to recover metastable sets from the computation of eigenfunctions. See in particular references [S, SFHD, HMS]. Let us emphasise that, using the bounds on equilibrium potentials obtained in Corollary 4.8 of [BEGK3], Theorem 1.3 implies that the eigenfunction corresponding to a minimum $x_{i}$ is exponentially close to a constant $\left(\sim e^{F\left(x_{i}\right) / 2 \epsilon}\right)$ in the connected component of the level set $\left\{y: F(y)<F\left(z^{*}\left(x_{i}, \mathcal{M}_{i-1}\right)\right)\right\}$ that contains $x_{i}$ (i.e. in the valley below the saddle point that connects $x_{i}$ to the set that lies below $x_{i}$ ), while it drops exponentially in the other connected components of the level set of this saddle; below the level of $x_{i}$ it is exponentially small in absolute terms. Note that this implies that the zeros of $\phi$ are generally not in the neighborhood of the saddle points, but much closer to the minima in $\mathcal{M}_{i-1}$. This fact was also observed in [HMS]. We would like to stress that the fact that the eigenfunctions drop sharply at the saddle points makes them very good indicators of the actual valley structure of the potential $F$, i.e. they become excellent approximations to the indicator functions of the metastable sets corresponding to the metastable exit time $1 / \lambda_{i}$.

Finally, it is almost a corollary from the results obtained above that metastable exit times are asymptotically exponentially distributed, when appropriate non-degeneracy conditions are met. 
Theorem 1.4. Assume that the Hessian of $F$ is non-degenerate at all local minima and saddle points. Let $x_{i}$ be a minimum of $F$ and let $D$ be any closed subset of $\mathbb{R}^{d}$ such that:

(i) if $\mathcal{M}_{i} \equiv\left\{y_{1}, \ldots, y_{k}\right\} \subset \mathcal{M}$ enumerates all those minima of $F$ such that $F\left(y_{j}\right) \leq$ $F\left(x_{i}\right)$, then $\bigcup_{j=1}^{k} B_{\epsilon}\left(y_{j}\right) \subset D$, and

(ii) dist $\left(z^{*}\left(x_{i}, \mathcal{M}_{i}\right), D\right) \geq \delta>0$ for some $\delta$ independent of $\epsilon$.

Assume further that the conditions of Theorem 1.2 are satisfied. Then there exists $\delta>0$ independent of $\epsilon$ and of $t$ such that for all $t>0$,

$$
\begin{aligned}
\mathbb{P}_{x_{i}}\left[\tau_{D}>t \mathbb{E}_{x_{k}} \tau_{D}\right]= & \left(1+O\left(e^{-\delta / \epsilon}\right)\right) e^{-t\left(1+O\left(e^{-\delta / \epsilon}\right)\right)} \\
& +\sum_{j>i} O\left(e^{-\delta / \epsilon}\right) e^{-t \lambda_{j} \mathbb{E}_{x_{i}} \tau_{D}}+O(1) e^{-t O\left(\epsilon^{d-1}\right) \mathbb{E}_{x_{i}} \tau_{D}} .
\end{aligned}
$$

The results of this paper together with those of [BEGK3] show that the methods to analyse metastable behaviour in discrete Markov chains introduced in [BEGK1, BEGK2] can be naturally extended to continuous diffusion processes. In particular we see that the metastable behaviour of continuous and discrete diffusions is virtually identical, and that all results for the discrete chains treated in [BEGK1] carry over to the corresponding diffusion approximations. In fact, our results in the diffusion case are sharper, since we were able to identify the constants in the prefactors of exponentially small or large terms (we expect, however, that with some extra work this improvement can also be carried over to the discrete chains, at least under certain conditions). There are a number of generalizations of these results that can be investigated: First, one can naturally consider diffusion processes on more general Riemannian manifolds. Second, one can consider extensions to locally infinitely divisible processes with mixed diffusion and jump components. Such extensions will require some extra work, but in principle our approach appears applicable, and qualitatively similar results should be obtainable. Another potentially interesting generalization concerns non-reversible diffusion processes. Here the main difficulty is the determination of the invariant measure, which our methods do not address at all. However, it is to be expected that at least in uniquely ergodic situations, some of our results can still be carried over. We hope to address these issues in future publications.

The remainder of this paper is organized as follows: In Section 2 we prove an a priori estimate on the spectrum of the generator when Dirichlet conditions are applied to small neighborhoods of all the local minima of $F$. In Section 3 we then show that the eigenvalues of the full generator are asymptotically close to those of the capacity matrix, which in turn are then evaluated in the generic situation. In the course of the proof we also identify the eigenvalues of the generator with the principle eigenvalues of appropriate Dirichlet operators. Finally, we derive from these results the exponential distribution of the mean exit times.

\section{A priori spectral estimates}

Most of the preparatory background and necessary technical a priori estimates were introduced in [BEGK3] and will be imported from there. In this section we give an additional a priori estimate on the spectrum of certain Dirichlet operators associated to $L_{\epsilon}$. More 
precisely, we derive a priori lower bounds on principal eigenvalues and for the Dirichlet problem in (regular) open sets $D \subset \Omega \subseteq \mathbb{R}^{d}$ with closure $\bar{D}$. We denote by $\partial D$ the boundary of $\bar{D}$. We denote by $\bar{\lambda}(D) \equiv \bar{\lambda}_{1}(D)$ the principal eigenvalue of the Dirichlet problem

$$
\begin{aligned}
\left(L_{\epsilon}-\lambda\right) f(x) & =0, \quad x \in D, \\
f(x) & =0, \quad x \in D^{c},
\end{aligned}
$$

where $D^{c} \equiv \mathbb{R}^{d} \backslash D$, and sometimes use the notation $L_{\epsilon}^{D^{c}}$ to indicate the Dirichlet operator corresponding to the problem 2.1.

The following lemma is a classical result of Donsker and Varadhan [DV]:

Lemma 2.1. The principal eigenvalue $\bar{\lambda}(D)$ satisfies

$$
\bar{\lambda}(D) \geq \frac{1}{\sup _{x \in D} \mathbb{E}_{x} \tau_{D^{c}}} .
$$

In the case when we consider diffusions on a compact set, Lemma 2.1 will yield a sufficiently good estimate. If $D$ is unbounded, the supremum on the right may be infinite and the estimate becomes useless. However, it is easy to modify the proof of Lemma 2.1 to yield an improvement.

Lemma 2.2. Let $\phi_{D}$ denote the normalized eigenfunction corresponding to the principal eigenvalue of $L_{\epsilon}^{D}$. Let $A \subset D$ be any compact set. Then

$$
\bar{\lambda}(D) \geq \frac{1}{\sup _{x \in A} \mathbb{E}_{x} \tau_{D^{c}}}\left(1-\int_{D \backslash A} d y e^{-F(y) / \epsilon}\left|\phi_{D}(y)\right|^{2}\right) .
$$

Moreover, for any $\delta>0$, there exists a bounded set $A \subset D$ (independent of $\epsilon$ ) such that

$$
\bar{\lambda}(D) \geq \frac{1}{\sup _{x \in A} \mathbb{E}_{x} \tau_{D^{c}}(1+\delta)}
$$

Proof. Let $w(x)$ denote the solution of the Dirichlet problem

$$
\begin{aligned}
L_{\epsilon} w(x) & =1, \quad x \in D, \\
w(x) & =0, \quad x \in D^{c} .
\end{aligned}
$$

Note that $w(x)=\mathbb{E}_{x} \tau_{D^{c}}$ (see e.g. Eq. (2.22) of [BEGK3]). Now,

$$
\begin{aligned}
\int_{D} d x e^{-F(x) / \epsilon} \phi(x)\left(L_{\epsilon} \phi\right)(x) & =\int_{D} d x e^{-F(x) / \epsilon} \nabla \phi(x) \cdot \nabla \phi(x) \\
& =\lim _{h \downarrow 0} h^{-2} \int_{D} d x e^{-F(x) / \epsilon} \sum_{i=1}^{d}\left(\phi\left(x+h e_{i}\right)-\phi(x)\right)^{2} .
\end{aligned}
$$

Since $a b \leq \frac{1}{2}\left(C a^{2}+b^{2} / C\right)$ for any $C>0$, taking $a=\phi\left(x+h e_{i}\right), b=\phi(x)$ and $C=w(x) / w\left(x+h e_{i}\right)$, one sees that

$$
\left(\phi\left(x+h e_{i}\right)-\phi(x)\right)^{2} \geq\left(\frac{\phi\left(x+h e_{i}\right)^{2}}{w\left(x+h e_{i}\right)}-\frac{\phi(x)^{2}}{w(x)}\right)\left(w\left(x+h e_{i}\right)-w(x)\right) .
$$


Inserting this inequality into 2.6 yields

$$
\begin{aligned}
\int_{D} d x e^{-F(x) / \epsilon} \phi(x)\left(L_{\epsilon} \phi\right)(x) & \geq \int_{D} d x e^{-F(x) / \epsilon} \frac{\phi(x)^{2}}{w(x)}\left(L_{\epsilon} w\right)(x) \\
& =\int_{D} d x e^{-F(x) / \epsilon} \frac{\phi(x)}{w(x)} \phi(x) \\
& \geq \frac{1}{\sup _{x \in A} w(x)} \int_{A} d x e^{-F(x) / \epsilon} \phi(x)^{2} .
\end{aligned}
$$

Choosing $\phi$ as the normalized eigenfunction with maximal eigenvalue yields 2.3.

We may assume without loss of generality that $F(x) \geq 0$ everywhere. We now claim that for any $\gamma>0$,

$$
\int d y e^{-\gamma \widetilde{F}(y) / \epsilon}\left|\phi_{D}(y)\right|^{2}<C_{\gamma}<\infty,
$$

where $\widetilde{F}(y) \equiv \min _{x \in \mathcal{M}}(F(y)-F(x))$. This clearly implies 2.4 .

To see this it is convenient to introduce $v(y) \equiv e^{-F(y) / 2 \epsilon} \phi_{D}(y)$, which is the corresponding ground-state eigenfunction of the operator

$$
H_{\epsilon} \equiv e^{-F(\cdot) / 2 \epsilon} L_{\epsilon} e^{F(\cdot) / 2 \epsilon}=-\epsilon \Delta+\frac{1}{4 \epsilon}|\nabla F(x)|^{2}-\frac{1}{2} \Delta F(x),
$$

which is a symmetric operator on $L^{2}(D, d y)$. The estimate 2.9 follows from a semiclassical Agmon estimate for the ground-state eigenfunction, $v$, that can be found in [HS1]. In our case this yields

$$
\int d y e^{(1-\gamma) \widetilde{F}(y) / \epsilon}|v(y)|^{2}<C_{\gamma}<\infty,
$$

which in turn implies 2.97. This completes the proof of the lemma.

We will first establish that $\bar{\lambda}(D)$ is at most polynomially small in $\epsilon$ if $D$ does not contain local minima, more precisely, define

$$
\mathcal{M}_{\epsilon} \equiv\{z \in \Omega: \operatorname{dist}(z, \mathcal{M}) \leq \epsilon\} .
$$

Remark. The choice of balls of radius $\epsilon$ is somewhat arbitrary, but works.

Lemma 2.3. Assume that $D \cap \mathcal{M}_{\epsilon}=\emptyset$. Then there is a finite positive constant $C$ such that

$$
\sup _{x \in D} \mathbb{E}_{x} \tau_{D^{c}} \leq C \sup _{x \in D}|\{y: F(y) \leq F(x)\}| \epsilon^{-d+1} .
$$

Proof. The starting point of the proof is the relation (which is an immediate consequence of [BEGK3], Eq. (2.27)])

$$
\int_{D} d y e^{-F(y) / \epsilon} h_{B_{\rho}(x), D^{c}(y)} \geq \inf _{z \in \partial B_{\rho}(x)} \mathbb{E}_{z} \tau_{D^{c} \operatorname{cap}_{B_{\rho}(x)}\left(D^{c}\right)}
$$


between mean time, equilibrium potential and capacities. It follows from the well known relation

$$
\mathbb{E}_{x} \tau_{D^{c}}=\int_{D} G_{D}(x, y) d y
$$

between mean time and Green function that the Harnack inequality of [BEGK3, Lemma 4.1] carries over to $\mathbb{E}_{z} \tau_{D^{c}}$, implying that, if $\rho=c \epsilon$, then

$$
\sup _{z \in \partial B_{\rho}(x)} \mathbb{E}_{z} \tau_{D^{c}} \leq C \inf _{z \in \partial B_{\rho}(x)} \mathbb{E}_{z} \tau_{D^{c}} .
$$

Combining this with 2.14) gives

$$
\sup _{z \in \partial B_{\rho}(x)} \mathbb{E}_{z} \tau_{D^{c}} \leq C \frac{\int_{D} d y e^{-F(y) / \epsilon} h_{B_{\rho}(x), D^{c}(y)}}{\operatorname{cap}_{B_{\rho}(x)}\left(D^{c}\right)} .
$$

We now distinguish the regions $\{y: F(y)>F(x)\}$ and $\{y: F(y) \leq F(x)\}$ in the integral. In the former, we just use the fact that $h_{B_{\rho}(x), D^{c}}(y) \leq 1$, while in the latter we invoke the upper bound from Proposition 4.3 in [BEGK3]. This gives

$$
\begin{aligned}
\sup _{z \in \partial B_{\rho}(x)} \mathbb{E}_{z} \tau_{D^{c}} & \leq C \frac{\int_{y \in D: F(y)>F(x)} d y e^{-F(y) / \epsilon}}{\operatorname{cap}_{B_{\rho}(x)}\left(D^{c}\right)} \\
& +C \frac{1}{\operatorname{cap}_{B_{\rho}(x)}\left(D^{c}\right)} \int_{y \in D: F(y) \leq F(x)} d y e^{-F(y) / \epsilon} \frac{\operatorname{cap}_{B_{\rho}(y)}\left(B_{\rho}(x)\right)}{\operatorname{cap}_{B_{\rho}(y)}\left(D^{c}\right)} .
\end{aligned}
$$

Using the upper and lower bounds on the capacities from Proposition 4.7 of [BEGK3], we get

$$
\begin{aligned}
\sup _{z \in \partial B_{\rho}(x)} \mathbb{E}_{z} \tau_{D^{c}} \leq & C^{\prime} \epsilon \rho^{-d+2} e^{+F(x) / \epsilon} \int_{y \in D: F(y)>F(x)} d y e^{-F(y) / \epsilon} \\
& +C^{\prime} \epsilon \rho^{-d+2} \int_{y \in D: F(y) \leq F(x)} d y .
\end{aligned}
$$

By our assumption on $F$, the first integral is bounded by a constant times $\exp (-F(x) / \epsilon)$ and the second is equal to the volume of the level set $\{F(y) \leq F(x)\}$. This implies the claimed bound.

Combining our results yields

Corollary 2.4. If $D \cap \mathcal{M}_{\epsilon}=\emptyset$, then there exists a finite positive constant $C<\infty$, independent of $\epsilon$, such that

$$
\bar{\lambda}(D) \geq C \epsilon^{d-1} .
$$

We can generalize the bounds obtained so far to sets $D$ containing some of the local minima of $F$. That is, let $\mathcal{N} \subset \mathcal{M}$ be non-empty and let

$$
\mathcal{N}_{\epsilon}=\left\{y \in \mathbb{R}^{d}: \operatorname{dist}(y, \mathcal{N}) \leq \epsilon\right\} .
$$

Assume that $D \supset \mathcal{N}_{\epsilon}$ and set

$$
A(x)=\left\{y: h_{B_{\epsilon}(x), D^{c} \backslash B_{\epsilon}(x)}(y)=\max _{y \in \mathcal{M}} h_{B_{\epsilon}(x), D^{c} \backslash B_{\epsilon}(x)}(y)\right\} .
$$

Then 
Lemma 2.5. Under the assumptions of Lemma 2.2

$$
\frac{1}{\bar{\lambda}(D)} \leq \sum_{i: x_{i} \in \mathcal{N}_{\epsilon}} \frac{\int_{A\left(x_{i}\right)} e^{-F(y) / \epsilon} d y}{\operatorname{cap}_{B_{\epsilon}\left(x_{i}\right)}\left(D \backslash B_{\epsilon}\left(x_{i}\right)\right)} .
$$

Proof. The proof is similar to that of the preceding corollary combined with the estimate on mean times given in Theorem 6.2 of [BEGK3]. We leave the details to the reader.

Remark. The key fact we need to extract from Lemma 2.5 is that

$$
\bar{\lambda}(D) \gtrsim \min _{i: x_{i} \in \mathcal{N}} e^{-\left[F\left(z^{*}\left(x_{i}, \mathcal{M}_{k}\right)-F\left(x_{i}\right)\right] / \epsilon\right]} .
$$

\section{Principal Dirichlet eigenvalues}

It is a well known fact that if $F$ has $n$ local minima, then $L_{\epsilon}$ has $n$ eigenvalues that are exponentially small in $\epsilon$ and that the next largest eigenvalue is of the order of a constant [FW, Kolo]. It is also known ([Kolo, Chapter 8, Proposition 2.2]) that the eigenspace of these eigenfunctions is exponentially close in the $L^{2}(\exp (-F(y) / \epsilon) d y)$-distance to the linear hull of the $n$ indicator functions $\chi_{i}$ of the attractors of the minima $x_{i}$ under the deterministic dynamical system $\dot{y}(t)=-\nabla F(y(t))$.

In this section and the next we will derive a precise characterization of these eigenvalues that together with the estimates on capacities of [BEGK3] will ultimately yield the exact asymptotic formulae of Theorem 1.2. This is the analogue of Section 4 of [BEGK2] for the diffusion case. Our approach can to some extent be seen as an application of the ideas of Wentzell's remarkable paper from 1973 [W2]. As we will see, the application of these ideas is not as straightforward as in the discrete case, but in principle very similar.

Before we turn to the details of this construction, it is useful to explain the general strategy.

Let us now consider a set of disjoint compact sets $B_{i} \equiv B_{\epsilon}\left(x_{i}\right), i=1, \ldots, k$. Let $\bar{\lambda}_{k}$ denote the principal eigenvalue of the Dirichlet operator $L_{\epsilon}$ with Dirichlet conditions on $\partial \mathcal{S}_{k} \equiv \bigcup_{i=1}^{k} \partial B_{i}$ (and on $\partial \Omega$, if this is not empty). Consider, for $\lambda<\bar{\lambda}_{k}$, the solution of the Dirichlet problem

$$
\begin{aligned}
\left(L_{\epsilon}-\lambda\right) f^{\lambda}(x) & =0, & & x \in \Omega \backslash \partial \mathcal{S}_{k}, \\
f^{\lambda}(x) & =\phi(x), & & x \in \partial \mathcal{S}_{k}, \\
f^{\lambda}(x) & =0, & & x \in \partial \Omega
\end{aligned}
$$

(i.e. we consider the Dirichlet problems in the exterior and the interior of the balls simultaneously; note that the principal eigenvalue of $L_{\epsilon}$ within a ball will always be larger than $\bar{\lambda}_{k}$ and so plays no rôle). In what follows, when specifying the Dirichlet problems the conditions of vanishing of the solution on the boundary of $\Omega$ will be understood and not mentioned anymore. The basic idea is now to construct an eigenfunction of the full operator $L_{\epsilon}$ as a solution of the problem (3.1) with suitably chosen $\phi$. Indeed, if $\lambda$ is an 
eigenvalue of $L_{\epsilon}$ and if we choose $\phi(x)$ as the eigenfunction corresponding to this eigenvalue, then $f^{\lambda}(x)$ is equal to $\phi$ everywhere. To see this, note that since $\phi(x)=f^{\lambda}(x)$ on $\partial \mathcal{S}_{k}$, for $x \in \mathcal{S}_{k}^{c}$ we have

$$
\left(L_{\epsilon}-\lambda\right)^{S_{k}}\left(f^{\lambda}-\phi\right)(x)=\left(L_{\epsilon}-\lambda\right)\left(f^{\lambda}-\phi\right)(x)=0 .
$$

But since $\lambda$ is not in the spectrum of $L_{\epsilon}^{\mathcal{S}_{k}}$, this implies that $f^{\lambda}(x)=\phi(x)$ on $\mathcal{S}_{k}^{c}$ as well. The same argument applies in the interior of $\mathcal{S}_{k}$. This means that $\lambda<\bar{\lambda}_{k}$ is an eigenvalue of $L_{\epsilon}$ if and only if we can find a function $\phi$ on $\partial \mathcal{S}_{k}$ such that the solution of the Dirichlet problem (3.1) is actually an eigenfunction of $L_{\epsilon}$ with eigenvalue $\lambda$. In other words, any eigenfunction corresponding to eigenvalues below the principal Dirichlet eigenvalue can be represented as a solution of (3.1).

Thus the eigenvalue problem reduces to finding out for which values of $\lambda$ for suitable $\phi$ on the boundaries of $B_{i},\left(L_{\epsilon}-\lambda\right) f^{\lambda}=0$ everywhere. In fact, $\left(L_{\epsilon}-\lambda\right) f^{\lambda}$ is in general a measure concentrated on the surface $\partial \mathcal{S}_{k}$; demanding that this surface measure be zero yields in general an integral equation for $\phi(x)$ on $\partial \mathcal{S}_{k}$, which is not particularly easy to handle. In the case of discrete Markov processes, we have considered a very similar problem in [BEGK2]. There, the balls $B_{i}$ were, however, simply the points $x_{i}$. The measure $\left(L_{\epsilon}-\lambda\right) f^{\lambda}$ was then a simple measure on the finite set $\mathcal{M}_{k}$, and the boundary condition reduced to the $k$ numbers $\phi\left(x_{i}\right)$, and the integral equation was reduced to a simple linear equation for the unknown vector $\phi\left(x_{i}\right), i=1, \ldots, k$. The condition for $\lambda$ to be an eigenvalue was thus simply that a certain determinant vanishes. It would be more than nice if we could reduce ourselves to a similarly simple condition in the present case. Indeed this would be so if we knew beforehand that $\phi(x)$ is constant on each surface $\partial B_{i}$. While this cannot be truly the case, if $\epsilon$ is small we may expect that $\phi$ varies little. In that case, we could, as we shall see, use perturbation arguments to arrive at the desired conclusion. Unfortunately, to obtain such control on eigenfunctions looks rather difficult. While the Harnack and Hölder inequalities will give us the desired control if we know that the eigenfunction does not change sign in a suitable neighborhood of the minimum, one cannot exclude that some minima are close to such zeros. We will see, however, that eigenfunctions do not change sign near a minimum, unless they are very small there. This will be enough to carry through our arguments.

We begin this program in this section with the somewhat simpler problem of the computation of the principal eigenvalues in domains $D \subset \Omega$. The main application will be later to the case where $D$ is $\Omega$ with some small balls around local minima of $F$ removed. This problem is considerably simpler because principle eigenfunctions are positive.

Regularity properties of harmonic functions. We first state a simple application of the Harnack and Hölder inequalities (see [GT, Corollaries 9.25 and 9.24]) that we have stated as Lemmata 4.1 and 4.2 in [BEGK3].

Lemma 3.1. Assume that $x$ is a local minimum of $F$. Let $\phi$ be a positive strong solution of $\left(L_{\epsilon}-\lambda\right) \phi=0,|\lambda| \leq 1$, on a ball $B_{4 \sqrt{\epsilon}}(x)$. Then there exists a constant $C<\infty$ and $\alpha>0$, both independent of $\epsilon$, such that

$$
\operatorname{osc}_{y \in B_{\epsilon}(x)} \phi(y) \leq C \epsilon^{\alpha / 2} \min _{y \in B_{\epsilon}(x)} \phi(x) .
$$


Proof. We can use Lemmata 4.1 and 4.2 stated in [BEGK3] with $\Lambda=\lambda=\epsilon, \gamma=1$, $c=\lambda$, and

$$
v=\epsilon^{-2} \sup _{y \in B_{4 \sqrt{\epsilon}}(x)}\|\nabla F(y)\|_{\infty}^{2} \leq \text { const } \cdot \epsilon^{-1} .
$$

Then, with $R=2 \sqrt{\epsilon}$, we deduce first from Lemma 4.2 of [BEGK3] that

$$
\sup _{y \in B_{2 \sqrt{\epsilon}}(x)} \phi(y) \leq C \inf _{y \in B_{2 \sqrt{\epsilon}}(x)} \phi(y)
$$

and then from Lemma 4.1 that

$$
\operatorname{osc}_{y \in B_{\epsilon}(x)} \phi(x) \leq C \epsilon^{\alpha / 2} \sup _{y \in B_{2 \sqrt{\epsilon}}(x)} \phi(y)\left(1+\sqrt{\epsilon}^{d+1}|\lambda|\right) .
$$

This implies the lemma if $\lambda$ is not too large.

Principal eigenvalues revisited. We will now improve on the estimates on principal eigenfunctions $\bar{\lambda}(D)$ obtained in Section 2 by showing that in the case when $D$ contains a local minimum of $F$, these estimates are essentially exact.

Proposition 3.2. Assume that $D$ contains $l \geq 1$ local minima of the function $F$ and that there is a single minimum $x \in D$ that realizes

$$
F\left(z^{*}\left(x, D^{c}\right)\right)-F(x)=\max _{i=1}^{l}\left[F\left(z^{*}\left(x_{i}, D^{c}\right)\right)-F\left(x_{i}\right)\right] .
$$

We write $B \equiv B_{\epsilon}(x)$. Then there exist $\alpha>0, C<\infty$, and $\delta>0$, independent of $\epsilon$, such that the principal eigenvalue $\bar{\lambda}(D)$ of the Dirichlet problem on D satisfies

$$
\frac{\operatorname{cap}_{B}\left(D^{c}\right)}{\left\|h_{B, D^{c}}\right\|_{2}^{2}}\left(1-C \epsilon^{\alpha / 2}\right)\left(1-e^{-\delta / \epsilon}\right) \leq \bar{\lambda}(D) \leq \frac{\operatorname{cap}_{B}\left(D^{c}\right)}{\left\|h_{B, D^{c}}\right\|_{2}^{2}}\left(1+C \epsilon^{\alpha / 2}\right)\left(1+e^{-\delta / \epsilon}\right)
$$

here and henceforth $\|\cdot\|_{2}$ denotes the $L^{2}$ norm with respect to the measure $e^{-F(y) / \epsilon} d y$.

Proof. Set $D^{0}=D \backslash B$. Then we know by Lemma 2.5 that there exists $\delta>0$ such that

$$
\bar{\lambda}\left(D^{0}\right) \geq e^{-\left[F\left(z^{*}\left(x, D^{c}\right)\right)-F(x)\right] / \epsilon} e^{\delta / \epsilon},
$$

while we know that $\bar{\lambda}(D)<\bar{\lambda}\left(D^{0}\right)$ (and expect $\bar{\lambda} \sim e^{-\left[F\left(z^{*}\left(x, D^{c}\right)\right)-F(x)\right] / e}$, i.e. much smaller). By the general philosophy outlined above, we know that the principal eigenfunction can be represented as the solution of the Dirichlet problem (both inside and outside $B$ )

$$
\begin{aligned}
\left(L_{\epsilon}-\lambda\right) f^{\lambda}(y) & =0, & & y \in D \backslash \partial B, \\
f^{\lambda}(y) & =\phi_{D}(y), & & y \in \partial B, \\
f^{\lambda}(y) & =0, & & y \in D^{c},
\end{aligned}
$$

where the boundary conditions $\phi_{D}$ are given by the actual principal eigenfunction. We will assume that $\operatorname{dist}\left(x, D^{c}\right) \geq \delta>0$, independent of $\epsilon$. Then $B_{4 \sqrt{\epsilon}}(x) \subset D$, and since 
$\phi_{D}$ is the principal eigenfunction, it may be chosen positive on $D$. Therefore Lemma 3.1 applies and shows that

$$
\inf _{y \in \partial B} \phi_{D}(y) \equiv c \leq \sup _{y \in \partial B} \phi_{D}(y) \leq\left(1+C \epsilon^{\alpha / 2}\right) c .
$$

We will normalize the eigenfunction such that $c=1$ Thus we can write $f^{\lambda}(x)=$ $h_{B, D^{c}}^{\lambda}(x)+\psi^{\lambda}(x)$, where $h_{B, D^{c}}^{\lambda} \equiv h^{\lambda}$ is the $\lambda$-equilibrium potential (see [BEGK3, Section 2]) that solves

$$
\begin{aligned}
& \left(L_{\epsilon}-\lambda\right) h^{\lambda}(y)=0, \quad y \in D \backslash \partial B, \\
& h^{\lambda}(y)=1, \quad y \in \partial B, \\
& h^{\lambda}(y)=0, \quad y \in D^{c},
\end{aligned}
$$

while $\psi^{\lambda}$ solves

$$
\begin{aligned}
\left(L_{\epsilon}-\lambda\right) \psi^{\lambda}(y) & =0, & & y \in D \backslash \partial B, \\
\psi^{\lambda}(y) & =\phi_{D}(y)-1, & & y \in \partial B, \\
\psi^{\lambda}(y) & =0, & & y \in D^{c} .
\end{aligned}
$$

We want that $\left(L_{\epsilon}-\lambda\right) f^{\lambda}(x)=0$ on all of $D$. Here we have to interpret $\left(L_{\epsilon}-\lambda\right) f^{\lambda}$ as a surface measure on $\partial B$. That is, if $g$ is a smooth test function that vanishes on $D^{c}$,

$$
\begin{aligned}
\int_{D} d y e^{-F(y) / \epsilon} g(y)\left(L_{\epsilon}-\lambda\right) f^{\lambda}(y) \equiv \int_{D} d y e^{-F(y) / \epsilon} f^{\lambda}(y)\left(L_{\epsilon}-\lambda\right) f^{\lambda}(y) g(y) \\
=\int_{D \backslash B} d y e^{-F(y) / \epsilon} f^{\lambda}(y)\left(L_{\epsilon}-\lambda\right) g(y)+\int_{\operatorname{int} B} d y e^{-F(y) / \epsilon} f^{\lambda}(y)\left(L_{\epsilon}-\lambda\right) g(y) \\
=\epsilon \int_{\partial B} e^{-F(y) / \epsilon}\left(g(y) \partial_{n(y)} f^{\lambda}(y)-f^{\lambda}(y) \partial_{n(y)} g(y)\right) d \sigma_{B}(y) \\
\quad+\epsilon \int_{\partial B} e^{-F(y) / \epsilon}\left(g(y) \partial_{-n(y)} f^{\lambda}(y)-f^{\lambda}(y) \partial_{-n(y)} g(y)\right) d \sigma_{B}(y) \\
=\epsilon \int_{\partial B} e^{-F(y) / \epsilon}\left(g(y) \partial_{n(y)} f^{\lambda}(y)+g(y) \partial_{-n(y)} f^{\lambda}(y)\right) d \sigma_{B}(y)
\end{aligned}
$$

where $d \sigma_{B}(y)$ denotes the Euclidean surface measure on $\partial B$, and $\partial_{ \pm n(y)}$ denote the normal derivatives at $y \in \partial B$ from the exterior and interior of $B$, respectively. Thus we can identify

$$
d y e^{-F(y) / \epsilon}\left(L_{\epsilon}-\lambda\right) f^{\lambda}(y)=\epsilon e^{-F(y) / \epsilon}\left(\partial_{n(y)} f^{\lambda}(y)+\partial_{-n(y)} f^{\lambda}(y)\right) d \sigma_{B}(y) .
$$

To get control on $\bar{\lambda}$, we can ask at least that the total mass of this measure on $\partial B$ vanishes, i.e. that

$$
0=\int_{\partial B} e^{-F(y) / \epsilon}\left(\partial_{n(y)} f^{\lambda}(y)+\partial_{-n(y)} f^{\lambda}(y)\right) d \sigma_{B}(y) .
$$

To evaluate this expression it will be convenient to observe that on $\partial B, h_{B, D^{c}}(y)=1$ for $y \in \partial B$ (where $h_{B, D^{c}} \equiv h_{B, D^{c}}^{\lambda=0}$ is the Newtonian potential; see [BEGK3, Section 2]). 
Moreover, on $B, h_{B, D^{c}}(y) \equiv 1$, so that $\partial_{-n(y)} h_{B, D^{c}}(y)$ vanishes on $\partial B$. Using these facts together with Green's second identity (see [BEGK3, Eq. (2.8)]), we get from [3.15] the condition

$$
\begin{aligned}
0= & \int_{\partial B} e^{-F(y) / \epsilon} \partial_{n(y)} h_{B, D^{c}}(y) f^{\lambda}(y)-\frac{\lambda}{\epsilon} \int_{D} d y e^{-F(y) / \epsilon} h_{B, D^{c}}(y) f^{\lambda}(y) \\
= & \int_{\partial B} e^{-F(y) / \epsilon} \partial_{n(y)} h_{B, D^{c}}(y)-\frac{\lambda}{\epsilon} \int_{D} d y e^{-F(y) / \epsilon} h_{B, D^{c}}(y) h_{B, D^{c}}^{\lambda}(y) \\
& +\int_{\partial B} e^{-F(y) / \epsilon} \partial_{n(y)} h_{B, D^{c}}(y) \psi^{\lambda}(y)-\frac{\lambda}{\epsilon} \int_{D} d y e^{-F(y) / \epsilon} h_{B, D^{c}}(y) \psi^{\lambda}(y) .
\end{aligned}
$$

(Note that the derivative $\partial_{n(y)}$ is in the direction of the interior of $B$.) The two terms involving $\psi^{\lambda}$ will be naturally treated as error terms. In fact, since $\partial_{n(y)} h_{B, D^{c}}(y)>0$, using Lemma 3.1, we get

$$
0 \leq \int_{\partial B} e^{-F(y) / \epsilon} \partial_{n(y)} h_{B, D^{c}}(y) \psi^{\lambda}(y) \leq C \epsilon^{\alpha / 2} \int_{\partial B} e^{-F(y) / \epsilon} \partial_{n(y)} h_{B, D^{c}}(y) .
$$

If we define $\delta \psi^{\lambda}=\psi^{\lambda}-\psi^{0}$, we see that $\delta \psi^{\lambda}$ solves the Dirichlet problem

$$
\begin{aligned}
\left(L_{\epsilon}-\lambda\right) \delta \psi^{\lambda}(y) & =\lambda \psi^{0}(y), & & y \in D \backslash \partial B, \\
\delta \psi^{\lambda}(y) & =0, & & y \in \partial B, \\
\delta \psi^{\lambda}(y) & =0, & & y \in D^{c},
\end{aligned}
$$

and thus

$$
\delta \psi^{\lambda}(y)=\lambda\left(L_{\epsilon}^{D^{c} \cup B}-\lambda\right)^{-1} \psi^{0}(y)
$$

and so

$$
\left\|\delta \psi^{\lambda}\right\|_{2} \leq \frac{\lambda}{\bar{\lambda}\left(D^{0}\right)-\lambda}\left\|\psi^{0}\right\|_{2} .
$$

By the same argument we also have

$$
\left\|h_{B, D^{c}}^{\lambda}-h_{B, D^{c}}\right\|_{2} \leq \frac{\lambda}{\bar{\lambda}\left(D^{0}\right)-\lambda}\left\|h_{B, D^{c}}\right\|_{2} .
$$

On the other hand, by the Poisson kernel representation of $\psi^{0}$,

$$
\psi^{0}(z)=-\epsilon \int_{\partial B}\left(\phi_{D}(y)-1\right) \partial_{n(y)} G_{D \backslash B}(x, y) d \sigma_{B}(y),
$$

where $G_{D \backslash B}(x, y)$ denotes the Green function for the Dirichlet problem in $D \backslash B$ (see [BEGK3, Section 2]). Since the normal derivative of the Green function is negative on $\partial B$, we get

$$
0 \leq \psi^{0}(z) \leq C \epsilon^{\alpha / 2} h_{B, D^{c}}(z)
$$

With

$$
\epsilon \int_{\partial B} e^{-F(y) / \epsilon} \partial_{n(y)} h_{B, D^{c}}(y)=\operatorname{cap}_{B}\left(D^{c}\right)
$$


(3.17) implies that

$$
\begin{aligned}
& 0 \geq \operatorname{cap}_{B}\left(D^{c}\right)-\lambda\left\|h_{B, D^{c}}\right\|_{2}^{2}\left(1-C \epsilon^{\alpha / 2}\right)\left(1-\lambda /\left(\bar{\lambda}\left(D^{0}\right)-\lambda\right)\right), \\
& 0 \leq \operatorname{cap}_{B}\left(D^{c}\right)\left(1+C \epsilon^{\alpha / 2}\right)-\lambda\left\|h_{B, D}\right\|_{2}^{2} .
\end{aligned}
$$

This implies the claimed bound on $\bar{\lambda}(D)$. Note that while we have only used a necessary condition for $\bar{\lambda}(D)$, the fact that there must be such an eigenvalue implies that it actually lies within the bounds given by 3.26 .

Remark. In the case when several of the minima within $D$ satisfy (3.7) (i.e. if $D$ contains several minima that are "equally deep"), one has to remove balls $B_{\epsilon}\left(x_{i}\right)$ for each of these minima. Then one may proceed as before. The only difference is that now there appears one value $c_{i}$ for each of the minima that is yet to be determined. One sees that in such a case $\bar{\lambda}(D)$ is determined by a variational formula

$$
\bar{\lambda}(D)=\min _{c_{1}, \ldots, c_{l} \geq 0} \frac{\int_{D} e^{-F(y) / \epsilon}\left\|\nabla h\left(c_{1}, \ldots, c_{l}\right)\right\|_{2}^{2}}{\left\|h\left(c_{1}, \ldots, c_{l}\right)\right\|_{2}^{2}}\left(1+O\left(\epsilon^{\alpha / 2}, e^{-\delta / \alpha}\right)\right)
$$

where

$$
\begin{gathered}
L_{\epsilon} h\left(x_{1}, \ldots, c_{l}\right)(y)=0, \quad y \in D \backslash \bigcup_{i=1}^{l} \partial B_{\epsilon}\left(x_{i}\right), \\
h\left(c_{1}, \ldots, c_{l}\right)(y)=c_{i}, \quad y \in \partial B_{\epsilon}\left(x_{i}\right) .
\end{gathered}
$$

It is easy to see that the result differs only by a constant factor from that in the nondegenerate case stated in the proposition.

Uniform estimates on principal eigenfunctions. The proof of Proposition 3.2 has already provided us with an approximation for the principal eigenfunction, namely $h_{B, D^{c}}$. We have seen that in $L^{2}$ this approximation is good on the order $\epsilon^{\alpha / 2}$. We will now show that this approximation is also uniformly good.

Proposition 3.3. Under the hypothesis of Proposition 3.2. the principal eigenfunction, $\phi_{D}$, of $L_{\epsilon}^{D^{c}}$, normalized so that $\inf _{y \in \partial B} \phi_{D}=1$, satisfies

$$
h_{B, D^{c}}(y) \leq \phi_{D}(y) \leq h_{B, D^{c}}(y)\left(1+C \epsilon^{\alpha / 2}\right)\left(1+e^{-\delta / \epsilon}\right) .
$$

Proof. Let us first assume that $D$ is bounded. Set $\delta f^{\lambda}=f^{\lambda}-f^{0}$. Then $\delta f^{\lambda}$ satisfies the Dirichlet problem

$$
\begin{aligned}
L_{\epsilon} \delta f^{\lambda}(y) & =\lambda \psi^{\lambda}(y), & & y \in D \backslash \partial B, \\
\delta f^{\lambda}(y) & =0, & & y \in \partial B, \\
\delta f^{\lambda}(y) & =0, & & y \in D^{c} .
\end{aligned}
$$

Thus we can write

$$
\frac{\delta f^{\lambda}(y)}{h_{B, D^{c}}(y)}=\int_{D \backslash B} \frac{1}{h_{B, D^{c}}(y)} G_{D \backslash B}(y, z) h_{B, D^{c}}(z) \frac{\delta f^{\lambda}(z)}{h_{B, D^{c}}(z)} .
$$


Assume that $M \equiv \sup _{y \in D \backslash B} f^{\lambda}(y) / h_{B, D^{c}}(y)<\infty$. Then 3.31 together with 3.24 implies that

$$
\begin{aligned}
M & \leq 1+C \epsilon^{\alpha / 2}+\lambda M \sup _{y \in D \backslash B} \int_{D \backslash B} \frac{1}{h_{B, D^{c}}(y)} G_{D \backslash B}(y, z) h_{B, D^{c}}(z) \\
& =1+C \epsilon^{\alpha / 2}+\lambda M \sup _{y \in D \backslash B} \mathbb{E}_{y}\left[\tau_{B} \mid \tau_{B} \leq \tau_{D^{c}}\right] .
\end{aligned}
$$

Using the representation of the conditional mean time from Proposition 6.1 of [BEGK3], one shows that

so that

$$
\sup _{y \in D \backslash B} \mathbb{E}_{y}\left[\tau_{B} \mid \tau_{B} \leq \tau_{D^{c}}\right]=1 / \bar{\lambda}(D \backslash B)
$$

$$
M \leq \frac{1+C \epsilon^{\alpha / 2}}{1-\bar{\lambda}(D) / \bar{\lambda}(D \backslash B)} \leq\left(1+C \epsilon^{\alpha / 2}\right)\left(1+e^{-\delta / \epsilon}\right) .
$$

Since by construction $h_{B, D^{c}}(y) \leq \phi_{D}(y)$, the assertion of the proposition follows.

It remains to justify the assumption $M<\infty$. However, this is easy. First, $\phi_{D}$ is bounded and $C^{2}(D)$. Thus, $\phi_{D}(y) / h_{B, D^{c}}(y)$ may only diverge when $h_{B, D^{c}}(y) \downarrow 0$. However, since $h_{B, D^{c}}$ is harmonic and non-negative on the boundary, it is strictly positive on $D$ by the strong maximum principle. Thus its explosion can occur only at the boundary of $D$, where $h_{B, D^{c}}(y)$ tends to zero. Moreover, its normal derivative on $\partial D$ is strictly (and since $\bar{D}$ is compact, uniformly) positive (see e.g. [Tay, Section 5, Proposition 2.2]). Therefore $\phi_{D}(y) / h_{B, D^{c}}(y)$ remains bounded also when $y \rightarrow \partial D$.

Therefore the proposition is proven if $\bar{D}$ is compact.

In the non-compact case, we can obtain a similar result for the supremum over compact subsets $\Gamma \subset \mathbb{R}^{d}$, using the rapid decay of the Green function in regions where $F(y)$ is getting very large.

\section{Exponentially small eigenvalues and their eigenfunctions}

We now generalize the analysis from the previous section to the construction of all small eigenvalues of $L_{\epsilon}$. To do this we need first to establish some a priori estimates on the behavior of eigenfunctions near the local minima of $F$.

A priori estimates on eigenfunctions near local minima. For the analysis of harmonic functions that are not necessarily positive, we need an application of an estimate for subharmonic functions that allows relating the oscillation to the $L^{2}$ norm.

Lemma 4.1. Let $\phi$ be a strong solution of $\left(L_{\epsilon}-\lambda\right) \phi=0$ on a ball $B_{c \sqrt{\epsilon}}(x)$. Then there exists a constant $C<\infty$, independent of $\epsilon$, such that

$$
\operatorname{osc}_{B_{c \sqrt{\epsilon}}} \phi \leq C \epsilon^{-d / 4}\left(\int_{B_{2 c \sqrt{\epsilon}}}|\phi(x)|^{2} d x\right)^{1 / 2} .
$$

Proof. This is just a specialization of Theorem 9.20 in [GT] (that gives upper bounds on the supremum for subharmonic functions in terms of $p$-norms) to our case, choosing the balls involved in such a way that the constants are uniform in $\epsilon$. 
Our aim is now to show that in the vicinity of order $\sqrt{\epsilon}$, eigenfunctions corresponding to the exponentially small eigenvalues of $L_{\epsilon}$ either have a constant sign, or are irrelevantly small. That this should be the case is suggested by the following result that we cite (in slightly adapted form) from ([Kolo], Chapter 8, Proposition 2.2):

Proposition 4.2. Let $\phi$ be a normalized eigenfunction of $L_{\epsilon}$ corresponding to one of the $|\mathcal{M}|$ smallest eigenvalues. Let $\gamma<\hat{\gamma} \equiv \min _{x, y \in \mathcal{M}}(\widehat{F}(x, y)-F(y))$. Let $D_{i}$ be the set of points in $y \in \Omega$ such that the solution of the differential equation $\frac{d}{d t} y(t)=-\nabla F(y(t))$ with initial condition $y(0)=y$ converges to $x_{i} \in \mathcal{M}$. Then there exist constants $c_{i}$ such that

$$
\left\|\phi-\sum_{i} c_{i} \mathbb{1}_{D_{i}}\right\|_{2, \mu_{\epsilon}} \leq C \exp (-\gamma / \epsilon)
$$

for some constant $C \equiv C_{\gamma}<\infty$.

Remark. The proposition is stated in [Kolo] for smooth $V$, but it is easy to see that the proof carries through for $V \in C^{3}(\Omega)$.

Unfortunately this estimate is not quite enough to conclude that $\phi$ does not change sign near any minimum. We will show that this is the case if the contribution of $\phi$ coming from a neighborhood of a given minimum is significant. For $D \subset \Omega$, set $\|f\|_{2, \mu_{\epsilon}, D} \equiv$ $\left(\int_{D}|f(x)|^{2} \mu_{\epsilon}(d x)\right)^{1 / 2}$.

For a given eigenfunction $\phi$ define

$$
J \equiv\left\{j:\|\phi\|_{2, \mu_{\epsilon}, D_{j}} \geq \exp (-\gamma / 2 \epsilon)\right\} .
$$

Lemma 4.3. If $\phi$ is one of the eigenfunctions of Proposition 4.2, $j \in J$, then there exists a constant $c_{j}$, a finite, $\epsilon$-independent constant $C$ and a positive, $\epsilon$-independent constant $\alpha$, such that for all $x \in B_{\sqrt{\epsilon}}\left(x_{j}\right),\left|\phi(x)-c_{j}\right| \leq C \epsilon^{\alpha / 2} c_{j}$.

Proof. We will first show that the weighted $L^{2}$ estimate on the deviation of $\phi$ from a constant implies a local unweighted $L^{2}$ estimate on balls of radius $r \sim \sqrt{\epsilon}$ near the minima $x_{j}, j \in J$.

Note first that from 4.2 it follows that

$$
\left\|\phi-c_{j}\right\|_{2, \mu_{\epsilon}, D_{j}} \leq C \exp (-\gamma / \epsilon) .
$$

Set $\widehat{\phi}(x) \equiv \phi(x) /\|\phi\|_{2, \mu_{\epsilon}, D_{j}}$, and $\hat{c}_{j} \equiv c_{j} /\|\phi\|_{2, \mu_{\epsilon}, D_{j}}$. Then due to the definition of $J$, for this locally normalized function we get the estimate

$$
\left\|\widehat{\phi}-\hat{c}_{j}\right\|_{2, \mu_{\epsilon}, D_{j}} \leq C \exp (-\gamma / 2 \epsilon) .
$$

Note that this estimate is now unchanged if we add a constant to $F(x)$, so that we can pretend for the moment that $F\left(x_{i}\right)=0$. Let $R>0$ be such that $B_{R}\left(x_{j}\right) \in D_{j}$. Since $x_{j}$ is a quadratic minimum, there exists a finite positive constant $b$ such that $F(x) \leq b\left(x-x_{j}\right)^{2}$ for $x \in B_{R}\left(x_{j}\right)$. Hence $(4.5)$ implies in particular that

$$
\int_{B_{R}\left(x_{j}\right)}\left(\widehat{\phi}(x)-\hat{c}_{j}\right)^{2} d x \leq C e^{b R^{2} / \epsilon} \exp (-\gamma / 2 \epsilon) .
$$

Note that also

$$
\int_{B_{R}\left(x_{j}\right)}|\widehat{\phi}(x)|^{2} d x \leq e^{b R^{2} / \epsilon}\|\widehat{\phi}\|_{2, \mu_{\epsilon}, D_{j}}=e^{b R^{2} / \epsilon}
$$


Now let $x \in B_{\sqrt{\epsilon}}\left(x_{j}\right)$. Then Lemma 4.1 implies that

$$
\operatorname{osc}_{B_{2 \sqrt{\epsilon}}} \widehat{\phi} \leq C \epsilon^{-d / 4}
$$

for some (new) finite, $\epsilon$-independent constant $C$. Now we can use the Hölder estimate (Corollary 9.24 in [GT] as specialized in Lemma 4.1 of [BEGK3]), to deduce that for $r<\sqrt{\epsilon}$

$$
\operatorname{osc}_{B_{r}} \widehat{\phi} \leq C \epsilon^{-d / 4}\left(\frac{r}{\epsilon^{1 / 2}}\right)^{\alpha}
$$

for a new constant and $\alpha>0$, independent of $\epsilon$. If we choose $r \sim \epsilon^{d / 4 \alpha+1}$, we can achieve that $\operatorname{osc}_{B_{r}(x)} \leq C \epsilon^{\alpha / 2}<\hat{c}_{i} / 2$ if $\epsilon$ is small enough. But by the estimate 4.6 , then $\widehat{\phi}$ must be close to $c_{j}$, uniformly on $B_{r}(x)$. Since this argument holds for all $x \in B_{\sqrt{\epsilon}}\left(x_{j}\right)$, we have $\left|\widehat{\phi}-\hat{c}_{j}\right| \leq C \epsilon^{\alpha / 2}$ in this ball.

Remark. We will see later that this estimate grossly overestimates the possible fluctuations of $\phi$.

Lemma 4.1 is also the appropriate tool to show that near the minima where the $L^{2}$ norm is very small, a similar estimate holds uniformly. Namely, we have

Lemma 4.4. Let $x_{i} \in \mathcal{M}, i \notin J$. Then

$$
\sup _{x \in B \sqrt{\epsilon}\left(x_{j}\right)}|\phi(x)| \leq C \epsilon^{-d / 2} e^{-\gamma / 2 \epsilon} e^{+F\left(x_{i}\right) / 2 \epsilon} .
$$

Proof. By Lemma 4.1.

$$
\begin{aligned}
& \sup _{x \in B_{\sqrt{\epsilon}}\left(x_{j}\right)}|\phi(x)| \leq C \epsilon^{-d / 4}\|\phi\|_{2, d x, B_{2 \sqrt{\epsilon}}\left(x_{j}\right)} \leq C^{\prime} \epsilon^{-d / 4}\|\phi\|_{2, \mu_{\epsilon}, B_{2 \sqrt{\epsilon}}\left(x_{j}\right)} \\
& \leq C^{\prime} \epsilon^{-d / 4} e^{+F\left(x_{i}\right) / 2 \epsilon}\|\phi\|_{2, \mu_{\epsilon}, D_{j}} \leq C^{\prime} \epsilon^{-d / 4} e^{-\gamma / 2 \epsilon} e^{+F\left(x_{i}\right) / 2 \epsilon},
\end{aligned}
$$

which proves the lemma.

Characterization of the eigenvalues. Let us now order all minima $x_{i}$ of $F$ in such a way that

$$
F\left(z^{*}\left(x_{i+1}, \mathcal{M}_{i}\right)\right)-F\left(x_{i+1}\right) \leq F\left(z^{*}\left(x_{i}, \mathcal{M}_{i-1}\right)\right)-F\left(x_{i}\right)
$$

for $i=1, \ldots, n-1$, where $\mathcal{M}_{i}=\left\{x_{1}, \ldots, x_{i}\right\}$. We put moreover $\mathcal{M}_{0} \equiv \Omega^{c}$. We also set $B_{i} \equiv B_{\epsilon}\left(x_{i}\right)$ and $\mathcal{S}_{i} \equiv \bigcup_{j=1}^{i} B_{i}$. Note that considerable simplifications occur when all inequalities in 4.12) are strict, and we will only consider this case here.

Suppose that we want to compute eigenvalues below $\bar{\lambda}\left(\Omega \backslash \mathcal{S}_{k}\right) \equiv \bar{\lambda}_{k}$. We know that if $\phi^{\lambda}$ is an eigenfunction with $\lambda<\bar{\lambda}_{k}$, then it can be represented as the solution of the Dirichlet problem

$$
\begin{aligned}
\left(L_{\epsilon}-\lambda\right) f^{\lambda}(y) & =0, & & y \in \Omega \backslash \partial \mathcal{S}_{k}, \\
f^{\lambda}(y) & =\phi^{\lambda}(y), & & y \in \partial \mathcal{S}_{k} .
\end{aligned}
$$

Thus, as in the analysis of principal eigenvalues above, the condition on $\lambda$ will be the existence of a non-trivial $\phi^{\lambda}$ on $\partial \mathcal{S}_{k}$ such that the surface measure

$$
d y e^{-F(y) / \epsilon}\left(L_{\epsilon}-\lambda\right) f^{\lambda}(y)=e^{-F(y) / \epsilon}\left(\partial_{n(y)} f^{\lambda}(y)+\partial_{-n(y)} f^{\lambda}(y)\right) d \sigma_{\mathcal{S}_{k}}(y)
$$

vanishes. A necessary condition for this to happen is of course the vanishing of the total 
mass on each of the surfaces $\partial B_{i}, i \leq k$, i.e.

$$
\int_{\partial B_{i}} e^{-F(y) / \epsilon}\left(\partial_{n(y)} f^{\lambda}(y)+\partial_{-n(y)} f^{\lambda}(y)\right) d \sigma_{\mathcal{S}_{k}}(y)=0 .
$$

In view of Lemmata 4.3 and 4.4 we have the following dichotomy: Let $c_{i}=\inf _{y \in B_{i}} \phi^{\lambda}(y)$. Then either

(i) $\sup _{y \in B_{i}}\left|\phi^{\lambda}(y) / c_{i}-1\right| \leq C \epsilon^{\alpha / 2}$, or

(ii) $\sup _{y \in B_{i}}\left|\phi^{\lambda}(y)\right| \leq C \epsilon^{-d / 4} e^{-\gamma / 2 \epsilon} e^{+F\left(x_{i}\right) / 2 \epsilon}$

We now consider all possible cases: Let $J \subset\{1, \ldots, k\}$ be the set of indices where (i) holds, and $J^{c} \equiv\{1, \ldots, k\} \backslash J$. Given such a partition, we set

$$
f^{\lambda}=\sum_{j \in J} c_{j}\left(h_{B_{j}, \mathcal{S}_{k} \backslash B_{j}}^{\lambda}+\psi_{j}^{\lambda}\right)+\sum_{i \in J^{c}} \psi_{i}^{\lambda},
$$

where the $h_{j}^{\lambda} \equiv h_{B_{j}, \mathcal{S}_{k} \backslash B_{j}}$ are the $\lambda$-equilibrium potentials (see [BEGK3, Section 2]), i.e. solutions of $\left(L_{\epsilon}-\lambda\right) h_{j}=O$ with boundary conditions 1 on $\partial B_{j}$ and 0 on $\partial\left(\mathcal{S}_{k} \backslash B_{j}\right)$.

Then $\psi_{j}^{\lambda}$ satisfies, for $j \in J$,

$$
\begin{aligned}
\left(L_{\epsilon}-\lambda\right) \psi_{j}^{\lambda}(y) & =0, & & y \in \Omega \backslash \partial \mathcal{S}_{k}, \\
\psi_{j}^{\lambda}(y) & =\phi^{\lambda}(y) / c_{j}-1, & & y \in \partial B_{j}, \\
\psi_{j}^{\lambda}(y) & =0, & & y \in \partial B_{i}, i \neq j,
\end{aligned}
$$

and for $j \in J^{c}$,

$$
\begin{aligned}
\left(L_{\epsilon}-\lambda\right) \psi_{j}^{\lambda}(y) & =0, & & y \in \Omega \backslash \partial \mathcal{S}_{k}, \\
\psi_{j}^{\lambda}(y) & =\phi^{\lambda}(y), & & y \in \partial B_{j}, \\
\psi_{j}^{\lambda}(y) & =0, & & y \in \partial B_{i}, i \neq j .
\end{aligned}
$$

We now proceed as in the analysis of principal eigenvalues, i.e. we write as necessary condition for $\lambda$ to be an eigenvalue that for all $i=1, \ldots, k$,

$$
\begin{aligned}
0= & \int_{\partial B_{i}} e^{-F(y) / \epsilon} h_{i}(y)\left(\partial_{n(y)} f^{\lambda}(y)+\partial_{-n(y)} f^{\lambda}(y)\right) d \sigma_{\partial \mathcal{S}_{k}}(y) \\
= & \int_{\partial \mathcal{S}_{k}} e^{-F(y) / \epsilon} \partial_{n(y)} h_{i}(y) f^{\lambda}(y) d \sigma_{\partial \mathcal{S}_{k}}(y)-\frac{\lambda}{\epsilon} \int d y e^{-F(y) / \epsilon} h_{i}(y) f^{\lambda}(y) \\
= & \sum_{j \in J} c_{j}\left[\int_{\partial B_{j}} e^{-F(y) / \epsilon} \partial_{n(y)} h_{i}(y)\left(1+\psi_{j}^{\lambda}(y)\right) d \sigma_{\partial \mathcal{S}_{k}}(y)\right. \\
& \left.-\frac{\lambda}{\epsilon} \int d y e^{-F(y) / \epsilon} h_{i}(y)\left(h_{j}^{\lambda}(y)+\psi_{j}^{\lambda}(y)\right)\right] \\
& +\sum_{j \in J^{c}}\left[\int_{\partial B_{l}} e^{-F(y) / \epsilon} \partial_{h(y)} h_{i}(y) \psi^{\lambda}(y) d \sigma_{\partial \mathcal{S}_{k}}(y)-\frac{\lambda}{\epsilon} \int d y e^{-F(y) / \epsilon} h_{i}(y) \psi_{j}^{\lambda}(y)\right] .
\end{aligned}
$$


Note that by the bounds (i) and (ii), we see that for $j \in J$,

$$
\begin{aligned}
& \left|\int_{\partial B_{j}} e^{-F(y) / \epsilon} \partial_{n(y)} h_{i}(y) \psi_{j}^{\lambda}(y) d \sigma_{\partial \mathcal{S}_{k}}(y)\right| \\
& \leq C \epsilon^{\alpha / 2}\left|\int_{\partial B_{j}} e^{-F(y) / \epsilon} \partial_{n(y)} h_{i}(y) d \sigma_{\partial} \mathcal{S}_{k}(y)\right|,
\end{aligned}
$$

and for $j \in J^{c}$,

$$
\begin{aligned}
& \left|\int_{\partial B_{j}} e^{-F(y) / \epsilon} \partial_{n(y)} h_{i}(y) \psi_{j}^{\lambda}(y) d \sigma_{\partial \mathcal{S}_{k}}(y)\right| \\
& \leq C \epsilon^{-d / 4} e^{-\gamma / 2 \epsilon} e^{F\left(x_{j}\right) / 2 \epsilon}\left|\int_{\partial B_{j}} e^{-F(y) / \epsilon} \partial_{n(y)} h_{i}(y) d \sigma_{\partial \mathcal{S}_{k}}(y)\right| .
\end{aligned}
$$

At this point it is convenient to realize that Green's first identity and the fact that the $h_{i}$ are harmonic imply that, for $i \neq j$,

$$
\begin{aligned}
\left|\int_{\partial B_{j}} e^{-F(y) / \epsilon} \partial_{n(y)} h_{i}(y) d \sigma_{B_{j}}(y)\right| & =\left|\int_{\partial B_{j}} e^{-F(y) / \epsilon} h_{j}(y) \partial_{n(y)} h_{i}(y) d \sigma_{B_{j}}(y)\right| \\
& =\epsilon^{-1}\left|\int_{\operatorname{ext}_{k}} d y e^{-F(y) / \epsilon}\left(\nabla h_{j}(y), \nabla h_{i}(y)\right)\right| \\
& \leq \epsilon^{-1} \sqrt{\operatorname{cap}_{B_{i}}\left(\mathcal{S}_{k} \backslash B_{i}\right) \operatorname{cap}_{B_{j}}\left(\mathcal{S}_{k} \backslash B_{j}\right)},
\end{aligned}
$$

where the last inequality uses the Cauchy-Schwarz inequality. Thus, for $j \in J \backslash\{i\}$,

$$
\begin{aligned}
& \mid \int_{\partial B_{j}} e^{-F(y) / \epsilon} \partial_{n(y)} h_{i}(y) \psi_{j}^{\lambda} d \sigma_{\partial \mathcal{S}_{k}}(y) \mid \\
& \leq C \epsilon^{\alpha / 2} \epsilon^{-1} \sqrt{\operatorname{cap}_{B_{i}}\left(\mathcal{S}_{k} \backslash B_{i}\right) \operatorname{cap}_{B_{j}}\left(\mathcal{S}_{k} \backslash B_{j}\right)}
\end{aligned}
$$

and for $j \in J^{c} \backslash\{i\}$,

$$
\begin{aligned}
& \left|\int_{\partial B_{j}} e^{-F(y) / \epsilon} \partial_{n(y)} h_{i}(y) \psi_{j}^{\lambda} d \sigma_{\partial \mathcal{S}_{k}}(y)\right| \\
& \leq C \epsilon^{-d / 4} e^{-\gamma / 2 \epsilon} e^{F\left(x_{j}\right) / 2 \epsilon} \epsilon^{-1} \sqrt{\operatorname{cap}_{B_{i}}\left(\mathcal{S}_{k} \backslash B_{i}\right) \operatorname{cap}_{B_{j}}\left(\mathcal{S}_{k} \backslash B_{j}\right)} .
\end{aligned}
$$

For the diagonal terms $i=j \in J$, this simplifies to

$$
\left|\int_{\partial B_{j}} e^{-F(y) / \epsilon} \partial_{n(y)} h_{i}(y) \psi_{j}^{\lambda} d \sigma_{\partial \mathcal{S}_{k}}(y)\right| \leq C \epsilon^{\alpha / 2} \operatorname{cap}_{B_{j}}\left(\mathcal{S}_{k} \backslash B_{j}\right) .
$$

For the remaining terms involving $\psi^{\lambda}$ in 4.19 , we conclude, in complete analogy to the derivation of the bounds (3.20) and (3.21), that for $j \in J$,

$$
\begin{aligned}
\int d y e^{-F(y) / \epsilon} h_{i}(y) & \left(h_{j}^{\lambda}(y)-h_{j}(y)+\psi_{j}^{\lambda}(y)\right) \\
= & O\left(\epsilon^{\alpha / 2}\right)\left(1+O\left(e^{-\delta / \epsilon}\right)\right) \int d y e^{-F(y) / \epsilon} h_{i}(y) h_{j}(y),
\end{aligned}
$$


and for $j \in J^{c}$,

$$
\int d y e^{-F(y) / \epsilon} h_{i}(y) \psi_{j}^{\lambda}(y)=O\left(\epsilon^{-d / 4} e^{-\gamma / 2 \epsilon} e^{F\left(x_{j}\right) / 2 \epsilon}\right) \int d y e^{-F(y) / \epsilon} h_{i}(y) h_{j}(y) .
$$

To control all off-diagonal terms, we still need to show that the normalized functions $h_{i}$ and $h_{j}$ are almost orthogonal.

Lemma 4.5. (i) There is a constant $C<\infty$ such that, for $i \neq j$,

$$
\int d y e^{-F(y) / \epsilon} h_{j}(y) h_{i}(y) \leq C \epsilon^{-(d+1) / 2} \min \left(e^{-\widehat{F}\left(x_{i}, \mathcal{S}_{k} \backslash B_{i}\right) / \epsilon}, e^{-\widehat{F}\left(x_{j}, \mathcal{S}_{k} \backslash B_{j}\right) / \epsilon}\right) .
$$

(ii) For all $i$,

$$
\int d y e^{-F(y) / \epsilon} h_{j}(y)^{2} \geq C \epsilon^{d / 2} e^{-F\left(x_{j}\right) / \epsilon}
$$

Proof. We first prove (i). For $i \neq j$,

$$
\begin{gathered}
\int d y e^{-F(y) / \epsilon} h_{j}(y) h_{i}(y)=\int_{y: F(y) \leq \max \left(\widehat{F}\left(x_{i}, \mathcal{S}_{k} \backslash B_{i}\right), \widehat{F}\left(x_{j}, \mathcal{S}_{k} \backslash B_{j}\right)\right)} d y e^{-F(y) / \epsilon} h_{j}(y) h_{i}(y) \\
+\int_{y: F(y)>\max \left(\widehat{F}\left(x_{i}, \mathcal{S}_{k} \backslash B_{i}\right), \widehat{F}\left(x_{j}, \mathcal{S}_{k} \backslash B_{j}\right)\right)} d y e^{-F(y) / \epsilon} h_{j}(y) h_{i}(y)
\end{gathered}
$$

In the second integral we just use the element $h_{i}(y) \leq 1$; by our general assumptions on $F$, this gives a bound $C e^{-\max \left(\widehat{F}\left(x_{i}, \mathcal{S}_{k} \backslash B_{i}\right), \widehat{F}\left(x_{j}, \mathcal{S}_{k} \backslash B_{j}\right)\right) / \epsilon}$. In the first integral we use the bounds on the equilibrium potential from Corollary 4.8 of [BEGK3]. Note that for any $y$, at most one of the factors $h_{i}(y)$ or $h_{j}(y)$ can be close to one. Thus even the roughest estimate yields ${ }^{2}$

$$
\begin{aligned}
& \int_{y: F(y) \leq \max \left(\widehat{F}\left(x_{i}, \mathcal{S}_{k} \backslash B_{i}\right), \widehat{F}\left(x_{j}, \mathcal{S}_{k} \backslash B_{j}\right)\right)} d y e^{-F(y) / \epsilon} h_{j}(y) h_{i}(y) \\
& \leq \int_{y: F(y) \leq \max \left(\widehat{F}\left(x_{i}, \mathcal{S}_{k} \backslash B_{i}\right), \widehat{F}\left(x_{j}, \mathcal{S}_{k} \backslash B_{j}\right)\right)} d y e^{-F(y) / \epsilon} \\
& \quad \times C \epsilon^{-1 / 2} e^{-\max \left(\widehat{F}\left(x_{i}, \mathcal{S}_{k} \backslash B_{i}\right), \widehat{F}\left(x_{j}, \mathcal{S}_{k} \backslash B_{j}\right)\right)} \\
& \leq C \epsilon^{-1 / 2}\left|\left\{y: F(y) \leq \max \left(\widehat{F}\left(x_{i}, \mathcal{S}_{k} \backslash B_{i}\right), \widehat{F}\left(x_{j}, \mathcal{S}_{k} \backslash B_{j}\right)\right)\right\}\right| \\
& \times e^{-\max \left(\widehat{F}\left(x_{i}, \mathcal{S}_{k} \backslash B_{i}\right), \widehat{F}\left(x_{j}, \mathcal{S}_{k} \backslash B_{j}\right)\right) / \epsilon} .
\end{aligned}
$$

Combining this upper bound with the lower bound we arrive at assertion (i).

To prove (ii), note that

$$
\begin{aligned}
\int d y e^{-F(y) / \epsilon} h_{j}(y)^{2} & \geq \int_{B_{\sqrt{\epsilon}}\left(x_{j}\right)} d y e^{-F(y) / \epsilon}\left(1-C \epsilon^{-1 / 2} e^{-\left[F\left(z^{*}\left(x_{j}, \mathcal{S}_{k} \backslash B_{j}\right)\right)\right] / \epsilon}\right)^{2} \\
& =C \epsilon^{d / 2} e^{-F\left(x_{j}\right) / \epsilon} .
\end{aligned}
$$

This concludes the proof of the lemma.

\footnotetext{
2 See the proof of 4.55 for more details.
} 
Let us define the capacity matrix ${ }^{3} \mathcal{C}$ with elements

$$
\mathcal{C}_{i j} \equiv \mathcal{C}_{i j}^{(k)} \equiv \epsilon \int_{\partial B_{j}} e^{-F(y) / \epsilon} h_{j}(y) \partial_{n(y)} h_{i}(y) d \sigma_{B_{j}}(y)
$$

and its normalized version

$$
\mathcal{K}_{i j} \equiv \mathcal{K}_{i j}^{(k)} \equiv \frac{\mathcal{C}_{i j}^{(k)}}{\left\|h_{i}\right\|_{2}\left\|_{j}\right\|_{2}} .
$$

Note that this matrix is symmetric and satisfies, by (4.22),

$$
\mathcal{K}_{i j} \leq \sqrt{\mathcal{K}_{i i} \mathcal{K}_{j j}}
$$

If we introduce the matrices

$$
\begin{aligned}
& A_{i j} \equiv \frac{\epsilon \int_{\partial B_{j}} e^{-F(y) / \epsilon} \partial_{n(y)} h_{i}(y) \psi_{j}^{\lambda}(y) d \sigma_{\partial \mathcal{S}_{k}}(y)}{\left\|h_{i}\right\|_{2}\left\|h_{j}\right\|_{2}} \\
& B_{i j} \equiv \begin{cases}\left(1-\delta_{i j}\right) \frac{\int d y e^{-F(y) / \epsilon} h_{i}(y)\left(h_{j}^{\lambda}(y)+\psi_{j}^{\lambda}(y)\right)}{\left\|h_{i}\right\|_{2}\left\|h_{j}\right\|_{2}} & \text { if } i \in J, \\
\left(1-\delta_{i j}\right) \frac{\int d y e^{-F(y) / \epsilon} h_{i}(y) \psi_{j}^{\lambda}(y)}{\left\|h_{i}\right\|_{2}\left\|h_{j}\right\|_{2}} & \text { if } j \in J^{c},\end{cases}
\end{aligned}
$$

and

$$
D_{i j} \equiv \delta_{i j} \frac{\int d y e^{-F(y) / \epsilon} h_{j}(y)\left(h_{j}^{\lambda}(y)-h_{j}(y)+\psi_{j}^{\lambda}\right)}{\left\|h_{j}\right\|_{2}^{2}},
$$

then the conditions (4.19) for $\lambda$ can be written as

$$
0=\sum_{j \in J} \hat{c}_{j}\left(\mathcal{K}_{i j}-\lambda \delta_{i j}+A_{i j}-\lambda\left(D_{i j}+B_{i j}\right)\right)+\sum_{j \in J^{c}}\left\|h_{j}\right\|\left(A_{i j}+\lambda B_{i j}\right),
$$

where $\hat{c}_{j}=\left\|h_{j}\right\|_{2} c_{j}$.

We can now collect the estimates on these matrix elements:

Lemma 4.6. The following bounds hold:

(i) For $i \neq j \in J$,

$$
\begin{aligned}
\left|B_{i j}\right| & \leq C \epsilon^{-d} \sqrt{\mathcal{K}_{i i} \mathcal{K}_{j j}}, \\
\left|D_{j j}\right| & \leq C \epsilon^{\alpha / 2}
\end{aligned}
$$

and for all $i, j$,

$$
\left|A_{i j}\right| \leq\left|\mathcal{K}_{i j}\right| C \epsilon^{\alpha / 2}
$$

\footnotetext{
3 The matrix $\mathcal{C}$ is a classical object in electrostatics, the diagonal elements being called capacities, and the off-diagonal ones coefficients of induction [Jack]. The off-diagonal coefficients represent the charge induced in the $i$-th ball when the $j$-th has potential one and all others are at potential zero.
} 
(ii) For $i \neq j \in J^{c}$,

$$
\begin{aligned}
\left\|h_{j}\right\|_{2}\left|A_{i j}\right| & \leq C \epsilon^{-3 d / 4}\left|\mathcal{K}_{i j}\right|, \\
\left\|h_{j}\right\|_{2}\left|B_{i j}\right| & \leq C \epsilon^{-d} e^{-\gamma / \epsilon} \sqrt{\mathcal{K}_{i i} \mathcal{K}_{j j}} .
\end{aligned}
$$

We collect the results obtained so far as

Theorem 4.7. Let $\mathcal{S}_{k} \equiv \bigcup_{i=1}^{k} B_{\epsilon}\left(x_{i}\right)$ and let $\bar{\lambda}_{k}$ denote the principal eigenvalue of the operator $L_{\epsilon}$ with Dirichlet conditions on $\partial \mathcal{S}_{k}$ (and $\partial \Omega$ ). Then a number $\lambda<\bar{\lambda}_{k}$ may be an eigenvalue of the operator $L_{\epsilon}$ if there exists a nonempty set $J \subset\{1, \ldots, k\}$, constants $c, \hat{c}_{j}, j \in J$, such that $\sum_{j \in J} \hat{c}_{j}^{2}=1$, and numbers $A_{i j}, B_{i j}, D_{i j}$ satisfying the constraints given in Lemma 4.6, such that for all $i \in J, E q$. 4.38) holds.

One would expect that Eq. (4.38) has a solution only when $\lambda$ is close to an eigenvalue of the matrix $\mathcal{K}$, and that indeed all eigenvalues of that matrix are close to the eigenvalues of $L_{\epsilon}$. We will not prove this directly. In fact, we will restrict our attention in this article to the non-degenerate situation when all "depths" of the valleys $x_{i}$ are distinct, i.e. when for all $i<k$ the inequalities 4.12 are strict.

Lemma 4.8. Let $\mathcal{K}_{i j}$ be the normalized capacity matrix and assume that

$$
\max _{i<k} \mathcal{K}_{i i} \leq e^{-\delta / \epsilon} \mathcal{K}_{k k}
$$

Then $k \in J$, and the largest eigenvalue, $\mu_{k}$, of $\mathcal{K}$ satisfies

$$
\mu_{k}=\mathcal{K}_{k k}\left(1+O\left(e^{-\delta / 2 \epsilon}\right)\right),
$$

while all other eigenvalues are smaller than $C e^{-\delta / \epsilon} \lambda_{k}$. Moreover, the eigenvector, $v=$ $\left(v_{1}, \ldots, v_{k}\right)$, corresponding to the largest eigenvalues normalized so that $v_{k}=1$ satisfies $\left|v_{i}\right| \leq C e^{-\delta / \epsilon}$ for $i<k$.

Proof. This is a simple perturbation argument. Note that we can write

$$
\mathcal{K}=\hat{\mathcal{K}}+\check{\mathcal{K}}
$$

where $\hat{\mathcal{K}}_{i j}=\mathcal{K}_{k k} \delta_{j k} \delta_{i k}$. Now we estimate the norm of $\check{\mathcal{K}}$ as in the proof of Lemma 4.5 .

Recall that

$$
\left|\mathcal{K}_{j i}\right| \leq \mathcal{K}_{i i} \mathcal{K}_{j j}
$$

Hence by assumption 4.44,

$$
\|\check{\mathcal{K}}\| \leq \mathcal{K}_{k k} \sqrt{e^{-\delta / \epsilon} k+e^{-\delta^{2} / \epsilon^{2}} k^{2}} .
$$

Since obviously $\hat{\mathcal{K}}$ has one eigenvalue $\mathcal{K}_{k k}$ with the obvious eigenvector and all other eigenvalues are zero, the announced result follows from standard perturbation theory.

Since $\mathcal{K}_{k k}=\operatorname{cap}_{B_{k}}\left(\mathcal{S}_{k-1}\right) /\left\|h_{k}\right\|_{2}^{2} \approx \bar{\lambda}_{k-1}$, this is precisely the value we expect. 
Corollary 4.9. Under the hypothesis (4.44), suppose that there exists an eigenvalue $\lambda_{k}$ of $L_{\epsilon}$ in the interval $\left(\bar{\lambda}_{k}, \bar{\lambda}_{k-1}\right]$.

(i) We have

$$
\lambda_{k}=\operatorname{cap}_{B_{k}}\left(\mathcal{S}_{k-1}\right) /\left\|h_{k}\right\|_{2}^{2}\left(1+O\left(\epsilon^{\alpha / 2}, e^{-\delta / \epsilon}\right)\right) .
$$

(ii) The eigenvalue $\lambda_{k}$ is simple and the corresponding eigenfunction $f_{k}^{\lambda}$ can be written as

$$
\phi_{k}^{\lambda}(y)=\frac{h_{k}(y)}{\left\|h_{k}\right\|_{2}}\left(1+O\left(\epsilon^{\alpha / 2}\right)\right)+\sum_{j=1}^{k-1} d_{j}(y) \frac{h_{j}(y)}{\left\|h_{j}\right\|_{2}},
$$

where $\left|d_{j}(y)\right| \leq e^{-\delta / \epsilon}$ for some $\delta>0$ (uniformly on compact subsets if $\Omega$ is unbounded).

Proof. First, if $k \notin J$, and if $\lambda_{k}$ is as assumed, then in each of the $|J|$ equations there is one term $\left(\mathcal{K}_{i i}+A_{i i}-\lambda\right) \hat{c}_{i} \sim \mathcal{K}_{k k} \hat{d}_{i}$ while all other terms are at most of order $\exp (-\delta / 2 \epsilon)\left|\mathcal{K}_{k k}\right| c_{j}$. Thus no normalized solution $\hat{c}$ can be found. Assume thus that $k \in J$. Considering all equations with $i \neq k$, the same argument as before shows that $\left|\hat{c}_{i}\right| \leq$ $C e^{-\delta / 2 \epsilon}$. Looking instead at the equation number $k$, since now $\hat{c}_{k} \approx 1$, it implies that

$$
\left|\left(\mathcal{K}_{k k}+A_{k k}-\lambda_{k}\right)\right| \leq C\left|\mathcal{K}_{k k}\right| e^{-\delta / \epsilon} \epsilon^{\alpha / 2},
$$

which yields (i).

As we have just seen that a solution of 4.38 with $\hat{c}_{k}=1$ must satisfy $\left|\hat{c}_{j}\right| \leq e^{-\delta / \epsilon}$ for all $j \neq k$, by (4.16), this implies that

$$
\phi_{k}^{\lambda}(y)=\frac{h_{k}^{\lambda}(y)+\phi_{k}^{\lambda}(y)}{\left\|h_{k}\right\|_{2}}+\sum_{j \in J \backslash k} \hat{c}_{j} \frac{h_{j}^{\lambda}(y)+\phi_{k}^{\lambda}(y)}{\left\|h_{j}\right\|_{2}} .
$$

Using the same arguments as in the proof of Proposition 3.3. and the bounds on $\phi^{\lambda}-c_{j}$ on the boundaries $\partial B_{j}$, we see that for $j \in J$,

$$
\begin{aligned}
\frac{\left|\phi_{j}^{\lambda}(y)-h_{j}(y)\right|}{\left\|h_{j}\right\|_{2}} & \leq C \epsilon^{\alpha / 2} \frac{h_{j}(y)}{\left\|h_{j}\right\|_{2}}+\sum_{l \in J_{j}} \frac{\operatorname{cap}_{B_{l}}\left(B_{j}\right)\left\|h_{l}\right\|_{2}}{\left\|h_{j}\right\|_{2}} \frac{h_{l}(y)}{\left\|h_{l}\right\|_{2}} \\
& \leq C \epsilon^{\alpha / 2} \frac{h_{j}(y)}{\left\|h_{j}\right\|_{2}}+\sum_{l \in J_{j}} e^{-\delta / \epsilon} \frac{h_{l}(y)}{\left\|h_{l}\right\|_{2}} .
\end{aligned}
$$

Combining these estimates we arrive at 4.50 . Note that this final estimate does not actually depend on the choice of $J$. Since two functions satisfying (4.50) cannot be orthogonal, it follows that $\lambda_{k}$ is a simple eigenvalue.

Now we can further explore the eigenvalues below $\bar{\lambda}_{k-1}$, etc., with the same results. Thus at the end of the procedure we arrive at the conclusion that $L_{\epsilon}$ can have at most the $n$ simple eigenvalues given by the values of the preceding corollary below the values $C \epsilon^{d-1}$. But since we know that there must be $n$ such eigenvalues, we conclude that all these candidates are in fact eigenvalues. This yields the following proposition: 
Proposition 4.10. Assume that all inequalities (4.12) are strict for all $i=1, \ldots, n$. Then the spectrum of $L_{\epsilon}$ below $\epsilon^{d-1}$ consists of $n$ simple eigenvalues that satisfy

$$
\begin{aligned}
\lambda_{k} & =\frac{\operatorname{cap}_{B_{k}}\left(\mathcal{S}_{k-1}\right)}{\left\|h_{k}\right\|_{2}^{2}}\left(1+O\left(\epsilon^{\alpha / 2}+e^{-\delta / \epsilon}\right)\right) \\
& =\operatorname{cap}_{B_{k}}\left(\mathcal{S}_{k-1}\right) \frac{\sqrt{\operatorname{det}\left(\nabla^{2} F\left(x_{k}\right)\right)}}{\sqrt{2 \pi \epsilon}^{d}} e^{F\left(x_{k}\right) / \epsilon}\left(1+O\left(\epsilon^{1 / 2}|\ln \epsilon|, \epsilon^{\alpha / 2}, e^{-\delta / \epsilon}\right)\right) \\
& =\frac{1}{\mathbb{E}_{x_{k}} \tau_{\mathcal{S}_{k-1}}}\left(1+O\left(\epsilon^{\alpha / 2}+e^{-\delta / \epsilon}\right)\right), \quad k=1, \ldots, n .
\end{aligned}
$$

The corresponding eigenfunctions satisfy 4.50 .

Proof. We have seen in fact that $\lambda_{k}=\mathcal{K}_{k k}^{(k)}\left(1+O\left(e^{-\theta / \epsilon}, \epsilon^{\alpha / 2}\right)\right)$, which provides the first assertion of Proposition 4.10 It remains to identify the eigenvalues with the inverse mean times. This follows from Proposition 6.1 in [BEGK3], provided we can show that

$$
\int d y e^{-F(y) / \epsilon} h_{k}(y)^{2} \sim \int d y e^{-F(y) / \epsilon} h_{k}(y) .
$$

In fact, we will show more, namely that both sides of (4.55) are asymptotically equal to

$$
e^{-F\left(x_{k}\right) / \epsilon} \frac{\sqrt{2 \pi \epsilon}^{d}}{\sqrt{\operatorname{det}\left(\nabla^{2} F\left(x_{k}\right)\right)}} .
$$

We must show that the main contribution of the integrals comes from a small neighborhood of $x_{k}$, which yields the contribution (4.56). It is clear that all contributions from the set $\left\{y: F(y)>F\left(x_{k}\right)+\epsilon|\ln \epsilon|\right\}$ give only sub-leading corrections. To treat the complement of this set, we use the bounds on the equilibrium potential of Eq. (4.27) in [BEGK3]. Up to polynomial factors in $\epsilon$, this implies that the integrand on the right-hand side of (4.55) (and a fortiori on the left-hand side) in the connected components of this level set that do not contain $x_{k}$ is smaller than

$$
e^{-\left[F(y)+F\left(z^{*}\left(y, B_{k}\right)\right)-F\left(z^{*}\left(y, \mathcal{S}_{k-1}\right)\right)\right] / \epsilon} .
$$

If $y$ is in the component of the level set that contains the minimum $x_{j}$, and $j<k$, we see that this is equal to

$$
e^{-F\left(z^{*}\left(x_{j}, B_{k}\right)\right) / \epsilon}
$$

which is exponentially smaller than $\exp \left(-F\left(x_{k}\right) / \epsilon\right)$, independent of $y$. If $j>k$, we still get the same result if $F(y) \geq F\left(z^{*}\left(x_{j}, \mathcal{S}_{k-1}\right)\right)$. Otherwise, we can write 4.57$]$ as

$$
e^{-\left[F(y)-F\left(x_{j}\right)\right] / \epsilon} e^{-\left[F\left(x_{k}\right)+\left(F\left(z^{*}\left(x_{j}, B_{k}\right)\right)-F\left(x_{k}\right)\right)-\left(F\left(z^{*}\left(x_{j}, \mathcal{S}_{k-1}\right)\right)-F\left(x_{j}\right)\right)\right] / \epsilon} .
$$

We will argue that

$$
F\left(z^{*}\left(x_{j}, B_{k}\right)\right)-F\left(x_{k}\right)>F\left(z^{*}\left(x_{j}, \mathcal{S}_{k-1}\right)\right)-F\left(x_{j}\right) .
$$

Assume the contrary. Note that trivially

$$
F\left(z^{*}\left(x_{j}, \mathcal{S}_{k-1}\right)\right) \geq F\left(z^{*}\left(x_{j}, \mathcal{S}_{j-1}\right)\right),
$$


while

$$
F\left(z^{*}\left(x_{j}, B_{k}\right)\right)=F\left(z^{*}\left(x_{k}, B_{j}\right)\right) \leq F\left(z^{*}\left(x_{k}, \mathcal{S}_{j} \backslash B_{k}\right)\right) .
$$

Therefore, our assumption implies that

$$
F\left(z^{*}\left(x_{j}, \mathcal{S}_{j-1}\right)\right)-F\left(x_{j}\right) \leq F\left(z^{*}\left(x_{k}, \mathcal{S}_{j} \backslash B_{k}\right)\right)-F\left(x_{k}\right),
$$

which a moment's reflection shows to be in contradiction with the conditions (4.12) at stage $j$. In other words, if our assumption were true, then the set $B_{k}$ would yield the largest eigenvalue at stage $j$, i.e. it would have had to be labeled $B_{j}$. Thus 4.60 must hold.

Since by assumption the inequalities are strict (which is more than we need), it follows that indeed

$$
\int d y e^{-F(y) / \epsilon} h_{k}(y)=e^{-F\left(x_{k}\right) / \epsilon} \frac{\sqrt{2 \pi \epsilon} d}{\sqrt{\operatorname{det}\left(\nabla^{2} F\left(x_{k}\right)\right)}}\left(1+O\left(\epsilon^{1 / 2}|\ln \epsilon|\right)\right)
$$

and of course the same bound holds when $h_{k}$ is replaced by $h_{k}^{2}$. This concludes the proof of the theorem.

Improved error estimates. To conclude the proofs of Theorems 1.2 and 1.3 we only need to improve the error estimates. In the proofs of this section we have produced error terms from two sources: the exponentially small errors resulting from the perturbation around $\lambda=0$ and the non-perfect orthogonality of the functions $h_{i}$, and the much larger errors of order $\epsilon^{\alpha / 2}$ that resulted from the a priori control on the regularity of the eigenfunctions obtained from the Hölder estimate of Lemma 4.3 . In the light of the estimates obtained on the eigenfunctions these can now be improved successively (as in the proof of Theorem 3.1 of [BEGK3]). Notice first that the eigenfunction corresponding to the minimum $x_{k}$ is small enough at all the minima $x_{l}, l<k$, so that we can actually take $J=\{k\}$ and $J_{k}=\{1, \ldots, k-1\}$ in 4.17, 4.19). Then we know from Corollary 4.9 that

$$
\operatorname{osc}_{y \in B_{4 \sqrt{\epsilon}}\left(x_{k}\right)} \phi_{k}(y) \leq C \epsilon^{\alpha / 2} \sup _{y \in B_{4 \sqrt{\epsilon}}\left(x_{k}\right)} \phi_{k}(y),
$$

which improves the a priori estimate 3.5. Then the Hölder estimate stated in Lemma 4.1 of [BEGK3] gives the improvement

$$
\begin{aligned}
\operatorname{osc}_{y \in B_{\epsilon}\left(x_{k}\right)} \phi_{k}(y) & \leq C \epsilon^{\alpha / 2}\left(C \epsilon^{\alpha / 2}+\lambda_{k} \epsilon^{(d+1) / 2}\right) \sup _{y \in B_{4 \sqrt{\epsilon}}\left(x_{k}\right)} \phi_{k}(y) \\
& \leq C \epsilon^{\alpha} \sup _{y \in B_{4 \sqrt{\epsilon}}\left(x_{k}\right)} \phi_{k}(y)
\end{aligned}
$$

over the estimate 3.3 . This allows us to replace all errors of order $\epsilon^{\alpha / 2}$ by errors of order $\epsilon^{\alpha}$. This procedure can be iterated $m$ times to get errors of order $\epsilon^{m \alpha / 2}$ until these are as small as the exponentially small errors.

Finally, we would like to improve the precision with which we relate the eigenvalues to the inverse mean exit times. This precision is so far limited by the precision with which

$$
\mathbb{E}_{x_{k}} \tau_{\mathcal{S}_{k-1}} \approx \frac{\operatorname{cap}_{B_{k}}\left(\mathcal{S}_{k-1}\right)}{\left\|h_{k}\right\|_{2}}
$$


holds. From Proposition 6.1 of [BEGK3] we know that this precision is limited only by the variation of $\mathbb{E}_{x} \tau_{\mathcal{S}_{k-1}}$ on $B_{k}$. To improve this, we need to control

$$
\frac{\operatorname{cap}_{B_{k}}\left(\mathcal{S}_{k-1}\right)}{\left\|h_{k}\right\|_{2}}-\frac{\operatorname{cap}_{B_{\epsilon}(x)}\left(\mathcal{S}_{k-1}\right)}{\left\|h_{B_{\epsilon}(x), \mathcal{S}_{k-1}}\right\|_{2}} .
$$

Now it is very easy to see that if $x \in B_{\sqrt{\epsilon}}\left(x_{k}\right)$, then

$$
\left|h_{B_{\epsilon}(x), \mathcal{S}_{k-1}}(y)-h_{k}(y)\right| \leq e^{-\delta / \epsilon} h_{k}(y) .
$$

Namely,

$$
\begin{aligned}
\left|h_{B_{\epsilon}(x), \mathcal{S}_{k-1}}(y)-h_{k}(y)\right| \leq & \mathbb{P}_{y}\left[\left\{\tau_{B_{k}}<\tau_{\mathcal{S}_{k-1}}\right\} \cap\left\{\tau_{\mathcal{S}_{k-1}}<\tau_{B_{\epsilon}(x)}\right\}\right] \\
& +\mathbb{P}_{y}\left[\left\{\tau_{B_{\epsilon}(x)}<\tau_{\mathcal{S}_{k-1}}\right\} \cap\left\{\tau_{\mathcal{S}_{k-1}}<\tau_{B_{k}} \tau_{B_{\epsilon}(x)}\right\}\right] .
\end{aligned}
$$

But by the Markov property

$$
\begin{aligned}
\mathbb{P}_{y}\left[\left\{\tau_{B_{k}}<\tau_{\mathcal{S}_{k-1}}\right\} \cap\left\{\tau_{\mathcal{S}_{k-1}}<\tau_{B_{\epsilon}(x)}\right\}\right] \\
\quad \leq \mathbb{P}_{y}\left[\tau_{B_{k}}<\tau_{\mathcal{S}_{k-1}}\right] \max _{z \in B_{k}} \mathbb{P}\left[\tau_{\mathcal{S}_{k-1}}<\tau_{B_{\epsilon}(x)}\right] \leq e^{-\delta / \epsilon} \mathbb{P}_{y}\left[\tau_{B_{k}}<\tau_{\mathcal{S}_{k-1}}\right] .
\end{aligned}
$$

The second summand in (4.70) is bounded in the same way.

This implies of course that

$$
\left\|h_{B_{\epsilon}(x), \mathcal{S}_{k-1}}\right\|_{2}-\left\|h_{k}\right\|_{2} \leq e^{-\delta / \epsilon}\left\|h_{k}\right\|_{2} .
$$

We only need a similar estimate for capacities. While this may appear more difficult at first sight, we can take advantage of the fact that as long as $\bar{\lambda}\left(\left(B_{\epsilon}(x) \cup \mathcal{S}_{k-1}\right)^{c}\right) \gg \lambda_{k}$, we can replace $B_{k}$ in the proof of Proposition 4.10 without further changes by $B_{\epsilon}(x)$. Thus

$$
\lambda_{k}=\frac{\operatorname{cap}_{B_{\epsilon}(x)}\left(\mathcal{S}_{k-1}\right)}{\left\|h_{B_{\epsilon}(x), \mathcal{S}_{k-1}}\right\|_{2}^{2}}\left(1+O\left(e^{-\delta / \epsilon}\right)\right)=\frac{\operatorname{cap}_{B_{k}}\left(\mathcal{S}_{k-1}\right)}{\left\|h_{k}\right\|_{2}^{2}}\left(1+O\left(e^{-\delta / \epsilon}\right)\right),
$$

which implies together with (4.72) that

$$
\left|\operatorname{cap}_{B_{\epsilon}(x)}\left(\mathcal{S}_{k-1}\right)-\operatorname{cap}_{B_{k}}\left(\mathcal{S}_{k-1}\right)\right| \leq e^{-\delta / \epsilon} \operatorname{cap}_{B_{k}}\left(\mathcal{S}_{k-1}\right) .
$$

Based on (4.74) and (4.71), one can improve Proposition 6.1 of [BEGK3] iteratively as above to get

$$
\mathbb{E}_{x_{k}} \tau_{\mathcal{S}_{k}}=\frac{\operatorname{cap}_{B_{k}} \mathcal{S}_{k-1}}{\left\|h_{k}\right\|_{2}}\left(1+O\left(e^{-\delta / \epsilon}\right)\right)
$$

which together with the capacity estimate given in Theorem 1.1 of [BEGK3] implies the first equality of Theorem 1.2 Thus all error terms of order $\epsilon^{\alpha / 2}$ can be removed in 4.54 and (4.50), completing the proofs of Theorems 1.2 and Theorem 1.3

Exponential distribution of exit times. We conclude this section with a result that will imply Theorem 1.4 on the exponential distribution of exit times. Let $L_{\epsilon}^{D}$ denote the Dirichlet operator with Dirichlet conditions in $D$. To avoid confusion, we assume that 
$D=\mathcal{S}_{k-1}$. Note that Proposition 4.10 (and its improvement) also applies to the operator $L_{\epsilon}^{D}$, and if we denote by $\bar{\lambda}_{k}^{i}$ the $i$-th eigenvalue of $L_{\epsilon}^{D}$, we see that within our usual errors,

$$
\bar{\lambda}_{k}^{i} \sim \lambda_{k+i}
$$

for $i=1, \ldots, n-k$, and the corresponding eigenfunction $\phi_{k}^{i}$ satisfies

$$
\phi_{k}^{i}(y)=\frac{h_{k+i}(y)}{\left\|h_{k+i}\right\|_{2}}\left(1+O\left(e^{-\delta / \epsilon}\right)\right)+\sum_{j=k}^{n} d_{j}(y) h_{j}(y)\left\|h_{j}\right\|_{2}
$$

with $\left|d_{j}(y)\right| \leq e^{-\delta / \epsilon}$. Let us denote henceforth by $\bar{\phi}_{k}^{i}$ the corresponding normalized eigenfunctions (e.g. $\left.\left\|\bar{\phi}_{k}^{i}\right\|_{2}=1\right)$. Note that $\bar{\phi}_{k}^{i}=\phi_{k}^{i}\left(1+O\left(e^{-\delta / \epsilon}\right)\right)$, so in fact they can be represented in the same way as 4.77 with redefined $d_{j}$ satisfying the same bounds.

Denote by $P_{k-i}$ the projector on the subspace generated by $\phi_{k-1}^{i}$ and by $P_{\perp}$ the projector to the subspace orthogonal to $\operatorname{span}\left(\bar{\phi}_{k-1}^{1}, \ldots, \bar{\phi}_{k-1}^{n-k}\right)$. Note that

$$
\begin{aligned}
\mathbb{P}_{x_{k}}\left[\tau_{D}>T\right] & =\left(\delta_{x_{k}}, e^{-T L_{\epsilon}^{D}} \mathbb{1}_{D^{c}}\right) \\
& =\sum_{i=1}^{n-k}\left(\delta_{x_{k}}, e^{-T L_{\epsilon}^{D}} P_{k-i} \mathbb{1}_{D^{c}}\right)+\left(\delta_{x_{k}}, e^{-T L_{\epsilon}^{D}} P_{\perp} \mathbb{1}_{D^{c}}\right) \\
& =\sum_{i=1}^{n-k} e^{-\bar{\lambda}_{k-1}^{i} T} \bar{\phi}_{k-1}^{i}\left(x_{k}\right) \int_{D^{c}} d y e^{-F(y) / \epsilon} \bar{\phi}_{k-1}^{i}(y)+O\left(e^{-T \bar{\lambda}_{n}}\right) .
\end{aligned}
$$

Given the precise control on the eigenfunctions, it is not difficult to infer that

$$
\begin{aligned}
\mathbb{P}_{x_{k}}\left[\tau_{D}>T\right] & =\left(\delta_{x_{k}}, e^{-T L_{\epsilon}^{D}} \mathbb{1}_{D^{c}}\right) \\
& =\sum_{i=1}^{n-k} e^{-\bar{\lambda}_{k-1}^{i} T} \bar{\phi}_{k-1}^{i}\left(x_{k}\right) \int_{D^{c}} d y e^{-F(y) / \epsilon} \bar{\phi}_{k-1}^{i}(y)+O\left(e^{-T \bar{\lambda}_{n}}\right) .
\end{aligned}
$$

Now using 4.55, 4.56, we get

$$
\begin{aligned}
\bar{\phi}_{k-1}^{i}\left(x_{k}\right) \int_{D^{c}} d y & e^{-F(y) / \epsilon} \bar{\phi}_{k-1}^{i}(y) \\
= & h_{k-1+i}\left(x_{k}\right) \frac{\int_{D^{c}} d y e^{-F(y) / \epsilon} h_{k-1+i}(y)}{\left\|h_{k-1+i}\right\|_{2}^{2}} \\
& +\sum_{\left(j, j^{\prime}\right) \neq(k-1+i, k-1+i)} d_{j} d_{j^{\prime}} h_{j}\left(x_{k}\right) \frac{\int_{D^{c}} d y e^{-F(y) / \epsilon} h_{j^{\prime}}(y)}{\left\|h_{j}\right\|_{2}\left\|h_{j}\right\|_{2}} \\
= & h_{k-1+i}\left(x_{k}\right)\left(1+O\left(e^{-\delta / \epsilon}\right)\right) \\
& +\sum_{\left(j, j^{\prime}\right) \neq(k-1+i, k-1+i)} d_{j} d_{j^{\prime}} h_{j}\left(x_{k}\right) c_{j, j^{\prime}} e^{-\left[F\left(x_{j}^{\prime}\right)-F\left(x_{j}\right)\right] / 2 \epsilon} .
\end{aligned}
$$


Now if $j=k$, the term in the last sum is

$$
d_{k} d_{j^{\prime}} c_{k, j^{\prime}} e^{-\left[F\left(x_{j}^{\prime}\right)-F\left(x_{k}\right)\right] / 2 \epsilon} \leq e^{-\delta / \epsilon}
$$

since $F\left(x_{j^{\prime}}\right)>F\left(x_{k}\right)$ for $j^{\prime \prime}>k$; in all other cases,

$$
h_{j}\left(x_{k}\right) \approx e^{-\left[F\left(z^{*}\left(x_{j}, \mathcal{M}_{k+i} \backslash x_{j}\right)-F\left(x_{k}\right)\right)\right] / \epsilon}<e^{-\left[F\left(x_{j}\right)-F\left(x_{k}\right)\right] / \epsilon}
$$

so that

$$
h_{j}\left(x_{k}\right) e^{-\left[F\left(x_{j}^{\prime}\right)-F\left(x_{j}\right)\right] / 2 \epsilon}<e^{-\left[F\left(x_{j}\right)-F\left(x_{k}\right)\right] / 2 \epsilon} e^{-\left[F\left(x_{j}^{\prime}\right)-F\left(x_{k}\right)\right] / 2 \epsilon}<\epsilon^{-\delta / \epsilon} .
$$

This shows that

$$
\mathbb{P}_{x_{k}}\left[\tau_{D}>T\right]=e^{-\bar{\lambda}_{k-1} T}\left(1+O\left(e^{-\delta / \epsilon}\right)\right)+\sum_{i=2}^{n-k} e^{-\bar{\lambda}_{k-1}^{i}} O\left(e^{-\delta / \epsilon}\right)+O(1) e^{-T \bar{\lambda}_{k-1}^{n-k}}
$$

This proves Theorem 1.4

Acknowledgements. We thank an anonymous referee of [BEGK2] for drawing our attention to the paper [W2] by Wentzell. We also thank M. Eckhoff for participation in an early stage of this work. We also thank the referees of this paper for numerous helpful remarks that greatly helped to improve the presentation. A. Bovier thanks the EPFL and V. Gayrard the WIAS for hospitality and financial support that made this collaboration possible.

\section{References}

[BEGK1] Bovier, A., Eckhoff, M., Gayrard, V., Klein, M.: Metastability in stochastic dynamics of disordered mean-field models. Probab. Theory Related Fields 119, 99-161 (2001) Zbl 1012.82015 MR 2001k:82096

[BEGK2] Bovier, A., Eckhoff, M., Gayrard, V., Klein, M.: Metastability and low-lying spectra in reversible Markov chains. Comm. Math. Phys. 228, 219-255 (2002) Zbl 1010.60088 MR 1911735

[BEGK3] Bovier, A., Eckhoff, M., Gayrard, V., Klein, M.: Metastability in reversible diffusion processes I. Sharp asymptotics for capacities and exit times, J. Eur. Math. Soc. 6, 399424 (2004)

[BluGet] Blumenthal, R. M., Getoor, R. K.: Markov Processes and Potential Theory, Academic Press, New York (1968) Zbl 0169.49204 MR 41 \#9348

[BuMa1] Buslov, V. A., Makarov, K. A.: A time-scale hierarchy with small diffusion. Teoret. Mat. Fiz. 76, 219-230 (1988) (in Russian); English transl.: Theoret. Math. Phys. 76, 818-826 (1989) Zbl 0698.35076 MR 90a:58188

[BuMa2] Buslov, V. A., Makarov, K. A.: Life spans and least eigenvalues of an operator of small diffusion. Mat. Zametki 51, 20-31 (1992) (in Russian); English transl.: Math. Notes 51, 14-21 (1992) Zbl 0755.34086 MR 93c:34161

[D1] Davies, E. B.: Metastable states of symmetric Markov semigroups. I. Proc. London Math. Soc. 45, 133-150 (1982) Zbl 0498.47017 MR 84e:47056a

[D2] Davies, E. B.: Metastable states of symmetric Markov semigroups. II, J. London Math. Soc. 26, 541-556 (1982) Zbl 0527.47028 MR 84e:47056b 
[D3] Davies, E. B.: Spectral properties of metastable Markov semigroups. J. Funct. Anal. 52, 315-329 (1983) Zbl 0525.47030 MR 84k:47037

[DV] Donsker, M. D., Varadhan, S. R. S.: On the principal eigenvalue of second-order elliptic differential operators. Comm. Pure Appl. Math. 29, 595-621 (1976) Zbl 0356.35065 MR $54 \# 13336$

[Doo] Doob, J. L.: Classical Potential Theory and its Probabilistic Counterpart. Grundlehren Math. Wiss. 262, Springer, Berlin (1984) Zbl 0549.31001 MR 85k:31001

[FW] Freidlin, M. I., Wentzell, A. D.: Random Perturbations of Dynamical Systems. Springer, Berlin (1984) Zbl 0922.60006 MR 99h:60128

[GM] Gaveau, B., Moreau, M.: Metastable relaxation times and absorption probabilities for multidimensional stochastic systems. J. Phys. A: Math. Gen. 33, 4837-4850 (2000) Zbl 0974.60096 MR 2001i:82049

[GS] Gaveau, B., Schulman, L. S.: Theory of nonequilibrium first-order phase transitions for stochastic dynamics. J. Math. Phys. 39, 1517-1533 (1998)

[GT] Gilbarg, D., Trudinger, N. S.: Elliptic Partial Differential Equations of Second Order. 2nd ed., Grundlehren Math. Wiss. 224, Springer, Berlin (1983) Zbl 0562.35001 MR 86c:35035

[HKN] Helffer, B., Klein, M., Nier, F.: Quantitative analysis of metastability in reversible diffusion processes via a Witten complex approach. Preprint (2004)

[HN] Helffer, B., Nier, F.: Hypoellipticity and spectral theory for Focker-Planck operators and Witten Laplacians. Preprint 03-25, IRMR, to appear in Lecture Notes in Math., Springer (2003)

[HS1] Helffer, B., Sjöstrand, J.: J. Multiple wells in the semiclassical limit. I. Comm. Partial Differential Equations 9, 337-408 (1984) Zbl 0546.35053 MR 86c:35113

[HS2] Helffer, B., Sjöstrand, J.: Puits multiples en limite semi-classique. II. Interaction moléculaire. Symétries. Perturbation. Ann. Inst. H. Poincaré Phys. Théor. 42, 127-212 (1985) Zbl 0595.35031 MR 87a:35142

[HS3] Helffer, B., Sjöstrand, J.: Multiple wells in the semiclassical limit. III. Interaction through nonresonant wells. Math. Nachr. 124, 263-313 (1985) Zbl 0597.35023 MR 87i:35161

[HS4] Helffer, B., Sjöstrand, J.: Puits multiples en mécanique semi-classique. IV. Étude du complexe de Witten. Comm. Partial Differential Equations 10, 245-340 (1985) Zbl 0597.35024 MR 87i:35162

[HKS] Holley, R. A., Kusuoka, S., Stroock, S. W.: Asymptotics of the spectral gap with applications to the theory of simulated annealing. J. Funct. Anal. 83, 333-347 (1989) Zbl 0706.58075 MR 92d:60081

[HMS] Huisinga, W., Meyn, S., Schütte, Ch.: Phase transitions and metastability for Markovian and molecular systems. Ann. Appl. Probab. 14, 419-458 (2004) MR 2023026

[Jack] Jackson, J. D., Classical Electrodynamics. 2nd ed., Wiley, New York (1975) Zbl 0997.78500 MR 55 \#9721

[Ka] Kato, T.: Perturbation Theory for Linear Operators. 2nd ed., Grundlehren Math. Wiss. 132, Springer, Berlin (1976) Zbl 0342.47009 MR 53 \#11389

[Kolo] Kolokoltsov, V. N., Semiclassical Analysis for Diffusions and Stochastic Processes. Springer, Berlin (2000) Zbl 0951.60001 MR 2001f:58073

[KoMak] Kolokoltsov, V. N., Makarov, K. A., Asymptotic spectral analysis of a small diffusion operator and the life times of the corresponding diffusion process. Russian J. Math. Phys. 4, 341-360 (1996) Zbl 0912.58042 MR 99b:58228

[Ma] Mathieu, P.: Spectra, exit times and long times asymptotics in the zero white noise limit. Stoch. Stoch. Rep. 55, 1-20 (1995) 
[Mi] Miclo, L.: Comportement de spectres d'opérateurs de Schrödinger à basse température. Bull. Sci. Math. 119, 529-553 (1995) Zbl 0886.60055 MR 97f:60169

[S] Schütte, Ch.: Conformational dynamics: modelling, theory, algorithm, and application to biomolecules. Preprint SC 99-18, ZIB-Berlin (1999)

[SFHD] Schütte, Ch., Fischer, A., Huisinga, W., Deuflhard, P.: A direct approach to conformational dynamics based on hybrid Monte Carlo. J. Comput. Phys. 151, 146-168 (1999) Zbl 0933.65145 MR 2000d:92004

[Si1] Simon, B.: Semiclassical analysis for low lying eigenvalues. 1. Ann. Inst. H. Poincaré 120, 295-307 (1983) Zbl 0526.35027

[Si2] Simon, B.: Semiclassical analysis for low lying eigenvalues. 2. Ann. of Math. 120, 89-118 (1984) Zbl 0626.35070

[Szni] Sznitman, A.-S.: Brownian Motion, Obstacles and Random Media. Springer Monogr. Math., Springer, Berlin (1998) Zbl 0973.60003 MR 2001h:60147

[Tay] Taylor, M. E.: Partial Differential Equations. Basic Theory. Texts Appl. Math., Springer, Berlin (1996)

[W1] Wentzell, A. D.: On the asymptotic behaviour of the greatest eigenvalue of a second order elliptic differential operator with a small parameter in the higher derivatives. Soviet Math. Dokl. 13, 13-17 (1972) MR 0308573

[W2] Wentzell, A. D.: Formulas for eigenfunctions and eigenmeasures that are connected with a Markov process. Teor. Veroyatnost. i Primenen. 18, 3-29 (1973) (in Russian) 